\title{
Plasmas meet plasmonics
}

\section{Everything old is new again}

\author{
A.E. Rider ${ }^{1,2}$, K. Ostrikov ${ }^{1,2, a}$, and S.A. Furman ${ }^{1}$ \\ 1 Plasma Nanoscience Centre Australia (PNCA), CSIRO Materials Science and Engineering, P.O. Box 218, Lindfield, \\ 2070 New South Wales, Australia \\ 2 Plasma Nanoscience @ Complex Systems, School of Physics, The University of Sydney, 2006 New South Wales, Australia
}

Received 26 April 2012 / Received in final form 29 June 2012

Published online 4 September 2012

(C) The Author(s) 2012. This article is published with open access at Springerlink.com

\begin{abstract}
The term 'plasmon' was first coined in 1956 to describe collective electronic oscillations in solids which were very similar to electronic oscillations/surface waves in a plasma discharge (effectively the same formulae can be used to describe the frequencies of these physical phenomena). Surface waves originating in a plasma were initially considered to be just a tool for basic research, until they were successfully used for the generation of large-area plasmas for nanoscale materials synthesis and processing. To demonstrate the synergies between 'plasmons' and 'plasmas', these large-area plasmas can be used to make plasmonic nanostructures which functionally enhance a range of emerging devices. The incorporation of plasmafabricated metal-based nanostructures into plasmonic devices is the missing link needed to bridge not only surface waves from traditional plasma physics and surface plasmons from optics, but also, more topically, macroscopic gaseous and nanoscale metal plasmas. This article first presents a brief review of surface waves and surface plasmons, then describe how these areas of research may be linked through Plasma Nanoscience showing, by closely looking at the essential physics as well as current and future applications, how everything old, is new, once again.
\end{abstract}

\section{Introduction}

\subsection{Opening remarks}

The recent explosion in nanoscale science and technology research has led to increased interest in the field of plasmonics. With the wealth of exotic and exciting nanoplasmonic applications [1] that are now possible with the unparalleled level of control over nanostructure growth afforded by modern fabrication techniques it is easy to forget just how closely plasmonics is linked to traditional plasma physics, both theoretically and experimentally. Other comprehensive reviews have noted the important role played by plasma physics in the development and formulation of plasmonics [2]. However, the purpose of this article is to present a discussion of how plasmonics and plasma physics are linked, not only via their theoretical and physical origins, but also through modern nanofabrication techniques - with a particular emphasis on plasmaaided nanoscale synthesis and processing. This current link will be demonstrated with a focus on the idea of resonances - e.g., gaseous plasmas sustained by resonances will be linked to resonances of localised surface plasmons sustained in and around solids.

\footnotetext{
a e-mail: kostya.ostrikov@csiro.au
}

It is believed that a staggering $99 \%$ of the visible matter in the universe exists in a plasma state [3]. As shown in Figure 1a, this spans 32 orders of magnitude from the very small plasmas in metals relevant in plasmonics around the order of a nanometre to massive extragalactic objects [4] (e.g., double radio galaxies) of the order of $10^{23} \mathrm{~m}$. Figure $1 \mathrm{~b}$ narrows the focus to the types of terrestrial plasmas that are typically only found in a laboratory setting, demonstrating the relationship between gaseous plasmas and plasmonics through plasma nanoscience. In more detail, plasmonics (essentially plasmas in metals) is a way to exert a greater degree of control over photonicsrelated applications i.e., confining and guiding light over sub-wavelength scales through the use of nanostructures and thin films. Similarly, manipulating plasma resonance sustained discharges is a way to exert control over nanoelectronics by controlling the etching and growth parameters of the nanoscale components (such as nanostructures and thin films). The common link between all of these fields is plasma nanoscience. Hence, as a result of the nanoscale control over energy and matter that is possible in plasma nanoscience [5] (i.e. manipulating gaseous plasmas to create controlled nanoarrays), a clear link may be drawn between gaseous plasma physics and photonics. Moreover, it will be shown that the nature of collective phenomena can mathematically be scaled up and down as 


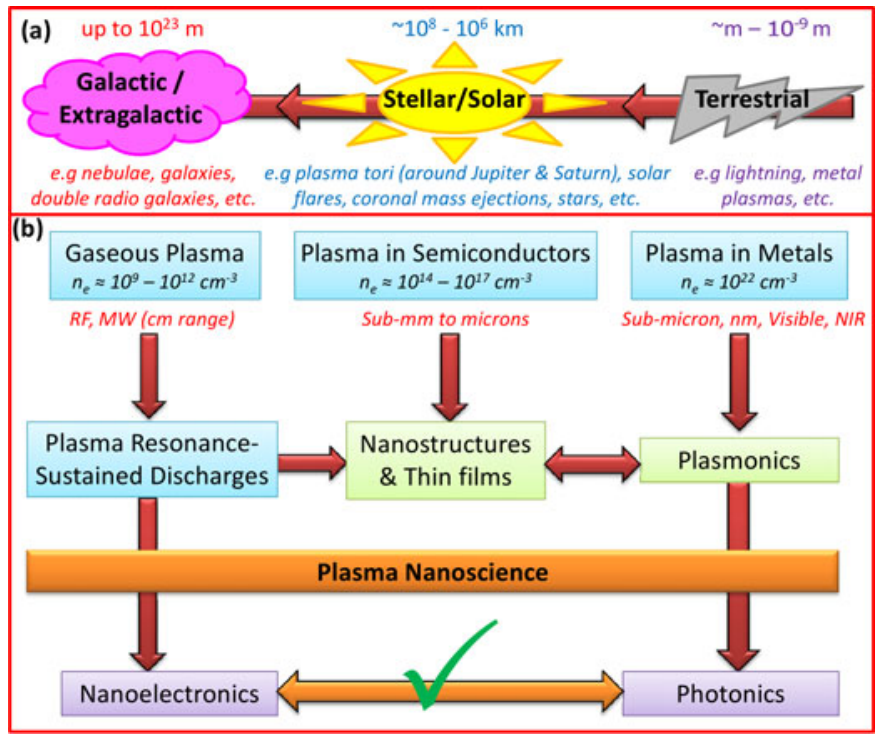

Fig. 1. (a) Typical sizes of plasmas - from extragalactic plasmas [4] to the metal plasmas of our interest. (b) Logic flowchart - from gaseous plasmas to nanoelectronics and photonics, where $n_{e}$ is the number density of electrons.

long as it is a plasma-from astrophysical plasmas to local surface plasmon resonances in metallic nanostructures.

The colloquium is organised as follows: for the rest of this Introduction we will provide some definitions, followed by a brief historical account of the most important milestones both in plasma physics and in plasmonics. Section 2 will then present the physics of surface waves, coming predominantly from a plasma physics perspective. This will be followed by a discussion of the physics of surface plasmons in Section 3 with an emphasis on optics. The two areas will be drawn together in Section 4 where the field of Plasma Nanoscience [6,7] will be used as a backdrop to discuss the ways in which plasmonics and plasma physics intersect.

\subsection{Definitions}

Before we can launch into a historical background or detailed treatment, given the multidisciplinary nature of plasmonics and the range of differing terminology used, a few definitions are necessary:

Plasmons are quasi-particles that are collective oscillations of conduction electrons in a material, excited by electromagnetic radiation [8]. They are also referred to as a 'quantized plasma (charge density) wave' [9]. These oscillations are similar to the electronic plasma oscillations in a gaseous discharge, which led to Pines coining the term "plasmon" to describe the phenomena in 1956 [10]. Three types of plasmons are commonly referred to in the literature:

Volume or Bulk plasmons are the cases discussed by Pines and Bohm [12-14], visualised in Figure 2. Here, a 'bulk plasmon' is the result of the creation of forward and

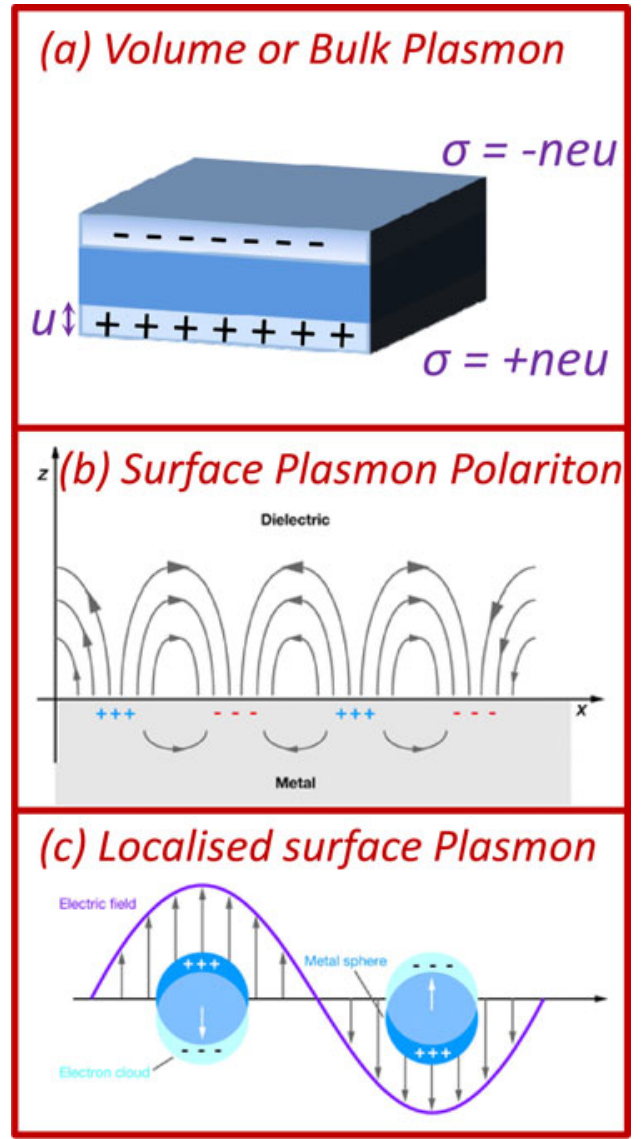

Fig. 2. Comparative sketches of (a) Volume plasmon (after Maier [11]); (b) surface Plasmon Polariton; (c) Localised surface plasmon. (b) and (c) reproduced with permission from [8], copyright 2007 Annual reviews.

backward electromagnetic (EM) waves (due to charge displacements caused by an incoming plane EM wave) - leading to the creation of an energy gap [2]. The energy of the plasmon is [9]:

$$
E_{b u l k}=\hbar \omega_{p}
$$

where $\omega_{p}$ is the plasma frequency, as derived by $H$. MottSmith [2]:

$$
\omega_{p}=\sqrt{\frac{4 \pi n_{e} e^{2}}{m_{e}}},
$$

where $n_{e}$ is the electron density, $e$ is the charge of an electron and $m_{e}$ is the effective mass of an electron. The dispersion relation of a transverse volume plasmon is [2]

$$
\omega^{2}=\omega_{p}^{2}+c^{2} k^{2}
$$

where $k$ is the wavenumber and $c$ is the speed of light. Hence, the phase and group velocities are given by:

$$
v_{p h}=\sqrt{\omega_{p}^{2}+c^{2} k^{2}} / k,
$$

and

$$
v_{g}=c^{2} k / \sqrt{\omega_{p}^{2}+c^{2} k^{2}},
$$

respectively. 
Surface plasmon polaritons (SPPs) also referred to as 'propagating surface plasmons' are a quantum of a polarised EM wave (called a polariton) in a propagating medium coupled with a plasmon [9] - depicted in Figure 2b. Assuming a perfect Drude free-electron model for the dielectric function, the energy of a SPP in a thin metal film in air is [9]:

$$
E_{S P P}=\hbar \omega_{p} / \sqrt{2}
$$

where $\omega_{p}$ is defined as in equation (2). Following on, the characteristic SPP frequency is [11]:

$$
\omega_{s p}=\frac{\omega_{p}}{\sqrt{1+\varepsilon_{2}}}
$$

where $\varepsilon_{2}$ is the real dielectric constant of the nonabsorbing dielectric half-space in a typical SPP set-up [11] which will be described in more detail in Section 1.3.

Localised surface plasmons (LSPs) arise when light hits a metal nanostructure. The light wavelength is much larger than the nanostructure, leading to a plasmon oscillating around the nanostructure [8] as depicted in Figure 2c. The energy of a surface plasmon in a small metallic sphere in air (again assuming Drude's model for the dielectric function) is [9]:

$$
E_{L S P}=\hbar \omega_{p} / \sqrt{3}
$$

Note a clear difference from equation (6), namely the use of $\sqrt{3}$ as opposed to $\sqrt{2}$. This is due to the effects of localisation (specifically, geometric confinement).

A surface wave plasma is "a bounded plasma" which "may support such (EM) waves which are guided along the boundary structure, their energy flux being concentrated in the vicinity of this surface" [15]. In short, the plasma guides the waves, which in turn provide energy to sustain the plasma. Simply, this is a type of plasma that is sustained by evanescently decaying electromagnetic surface waves, typically provided via a wave launcher such as a surfatron, surfaguide or microwave slot exciter/coupler. Initially, such plasmas were only possible in long, narrow tubes - which made them useful as a diagnostic tool, rather than a viable processing tool. However, recent developments have seen the generation of surface wave plasmas larger than $1 \mathrm{~m}^{2}$ (supported by a dielectric plate, rather than a tube), which makes them particularly promising for large-area processing.

Resonances are a phenomenon that are intrinsic to a bounded system. First discussed in the early 17th century by Galileo in conjunction with his work on pendulums presented in Dialogues concerning two sciences, they can be simply thought of as sympathetic vibrations or defined in more detail as "an enhancement of the response of a system to an external excitation at a particular frequency" [16]. The idea of resonances is one that is central to this colloquium and in demonstrating the link between plasma physics and plasmonics. For example, gaseous plasmas sustained by resonances as a means of nanofabrication of metallic or doped semiconductor nanomaterials which when excited by EM radiation at a specific frequency give rise to their own characteristic resonance frequencies which can be used in various devices, e.g., sensors, solar cells, etc. (see Fig. 1b).

\subsection{Historical background}

A comparison of the evolution of the plasma physics and optics fields, with particular plasmonic milestones marked, is presented in the timeline in Figure 3. Whilst the use of plasmons can be traced back to the 4th century AD (i.e., the Lycurgus cup, popularly in medieval stained glass windows thereafter), with further commentary by Faraday in 1857 [17], an understanding of what they are and how they worked was not truly established until the 20th century. The basis for treatment of light scattering by metals was largely set by the end of the first decade of the 1900's, with seminal works including Drude's treatment of metals in 1900 [18] and Mie's theory for scattering and absorption of electromagnetic radiation by a sphere in 1908 [19]. These works still form the theoretical background of the majority of papers on localised surface plasmons.

On the other hand, while terrestrial gas plasmas (e.g. lightning) has existed since primordial earth, experimental studies on the fourth state of matter did not occur until 1879 by Crookes, with its more common name plasma being coined by Langmuir in 1928 [31]. Langmuir and Tonks followed this up with the observation of electron plasma oscillations (also referred to as Langmuir oscillations) in 1929 [35]. The earliest work on plasma resonance sustained discharges [36] is that of Tonks $[32,37]$ who derived the resonance frequency of a plasma (in a cylindrical discharge tube) in 1931 [37]:

$$
\omega=\omega_{p} / \sqrt{2},
$$

where $\omega_{p}$ is defined as in equation (2).

The works of Zenneck [29] and Sommerfeld [30] from 1899-1909 on electromagnetic surface waves can be regarded as one of the first linkages between plasmas and plasmons, although neither were referred to as such at the time. These Sommerfeld-Zenneck waves (SZW) are similar to SPPs, the difference that being the condition for SZW is $\varepsilon_{i}(\omega) \gg\left|\varepsilon_{r}(\omega)\right|$ (where $\varepsilon_{i}$ and $\varepsilon_{r}$ are the imaginary and real parts of the dielectric function, respectively), whereas for SPPs it is $\varepsilon_{i}(\omega) \ll\left|\varepsilon_{r}(\omega)\right|$ [38], i.e., $\varepsilon_{r}$ must be of different signs for the two media for SPP, which is not the case for SZW. However, despite this, both fields existed separately for a long time with their common linkages not becoming clear until 1956 and 1957, first through the work of Pines [10] and then Ritchie [28], who defined plasmons (in general) and surface plasmons (specifically), respectively.

The defining paper on Fano resonances [39] was published in Physical Review in 1961, presenting a formulation for the asymmetrical resonance peaks observed in the absorption profiles of Rydberg spectral atomic lines [16]. Previously, resonances had been considered to be totally 


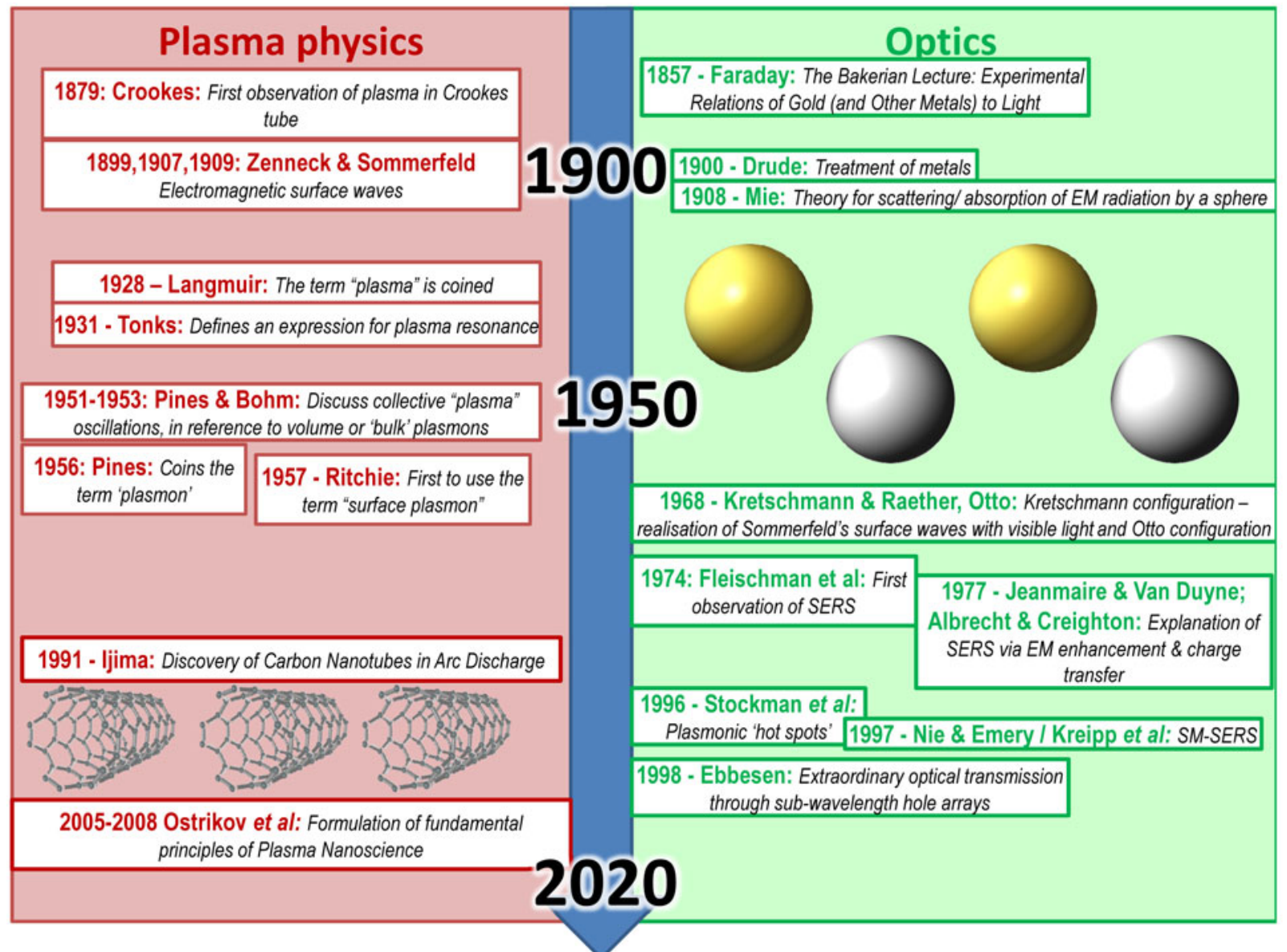

Fig. 3. Historical timeline including relevant milestones in the fields of optics [17-27] and plasma physics [6,10,12-14,28-34] from 1857 to the present day, discussed in detail in Section 1.3 of the main text.

symmetrical peaks, i.e., Lorentzian [40]. Fano resonances have been used in local surface plasmon resonance (LSPR) sensors [40] and will be discussed in more detail in Section 4.3.

Other relevant breakthroughs in the area include the development of the Otto [21] and Kretschmann configurations for the excitation of SPPs [20], the latter being the first experimental realisation of Sommerfelds surface waves with visible light; both configurations debuting in 1968. The difference between the configurations is that the Otto set-up has an air gap between the metal film and the prism (see Fig. 4d), as well as a thicker metal film ( $\sim 100$ 's of $\mathrm{nm}$ vs. $\sim 50 \mathrm{~nm}$ for the Kretschmann configuration). The Kretschmann configuration (see Fig. 4e) is still being used in modern surface plasmon resonance sensing experiments. The Kretschmann angle, $\theta_{K}$ is the angle at which SPPs can be excited [41] and is defined via:

$$
\sqrt{\varepsilon_{1}} \sin \theta_{K}=\sqrt{\frac{\varepsilon_{m} \varepsilon_{2}}{\varepsilon_{m}+\varepsilon_{2}}}
$$

where $\varepsilon_{1}$ and $\varepsilon_{2}$ are the permitivities of the glass prism and the medium in the half plane above the metal film, respectively, and $\varepsilon_{m}$ is the permittivity of the metal film in Kretschmann configuration.
The theme of sensing is of particular importance to plasmonics - not only through plasmon resonance spectroscopy, but also via Surface Enhanced Raman Scattering (SERS). The SERS phenomenon was first observed by Fleischman et al. [22] in 1974 and explained in 1977 as resulting from an electromagnetic enhancement mechanism by Jeanmaire and Van Duyne [24] and as due to a chemical enhancement mechanism (charge transfer) by Albrecht and Creighton [23] - also in 1977. Both theories persist to this day, appearing to be complementary, rather than exclusive interpretations of the SERS phenomena.

Debate about the origin of the SERS effect, however, is still ongoing. Surface enhanced Raman-based sensors employ a rough metal surface as a SERS substrate. Typical examples include roughened noble metal films (e.g., Ag was used by Fleischman et al. [22]) and more recently, nanostructured surfaces such as nanoparticle/nanodisk/nanotriangle arrays, etc. When the metal nanostructures are irradiated by a laser, which is in resonance with the characteristic frequency of the material (i.e., $633 \mathrm{~nm}$ for bulk Au), plasmons are generated around the nanostructure, which give rise to a significant enhancement in the local electric field around the nanostructure, which in turn amplifies the Raman modes of the molecule 


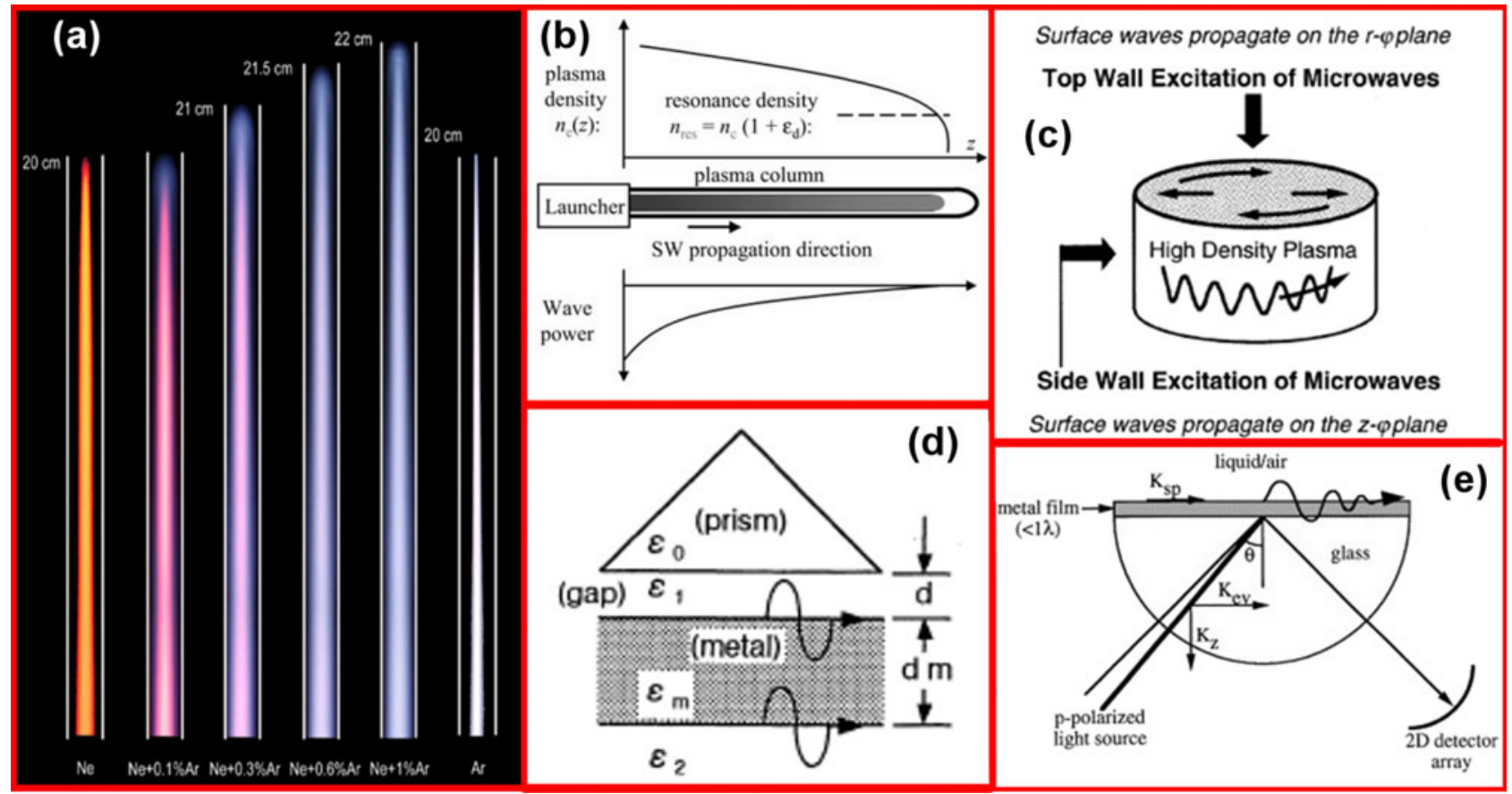

Fig. 4. (a) Surface wave tubular discharges, reproduced with permission from [44], IOP Publishing Ltd.; (b) generation of linear surface wave discharge and relation of number density to resonance, reproduced with permission from [45], IOP Publishing Ltd.; (c) reactor schematic for production of large area surface wave plasmas, reproduced with permission from [46], IOP Publishing Ltd., (d) Otto configuration for excitation of surface plasma waves - from [47], Copyright 1997 The Japan Society of Applied Physics. (e) Kretschmann configuration for excitation of SPPs used in SPR, reprinted from [48], with permission from Elsevier.

being studied. Hence, it may be recognised that SERS is in essence, due to its reliance on plasmons, a plasma effect.

An expression for the SERS enhancement factor is given by [8]:

$$
\operatorname{EF}_{S E R S}\left(\omega_{\nu}\right)=\frac{\left[I_{S E R S}\left(\omega_{\nu}\right) / N_{\text {surf }}\right]}{\left[I_{N R S}\left(\omega_{\nu}\right) / N_{v o l}\right]},
$$

where $I_{S E R S}$ is the intensity of the SERS signal, $I_{N R S}$ is the intensity of the normal Raman signal, $N_{\text {surf }}$ is the number of molecules bound to the SERS substrate and $N_{v o l}$ is the number of molecules in the excitation volume [8]. The intensity of the SERS signal, $I_{S E R S}$ can be expressed as $M\left(\omega_{L}\right) M\left(\omega_{R}\right) I_{N R S}$ [42], where $M(\omega)$ is the field intensity enhancement due to plasmon resonances and $\omega_{L}$ and $\omega_{R}$ are the exciting laser frequency and emitted photon frequency, respectively [42]. Hence, $I_{S E R S}$ can be regarded as due to a plasma effect of plasmon resonance.

An extension is single-molecule SERS sensors [43] which harnesses plasmonic hot spots (resulting from coupling between individual nanostructures) to detect ultra low concentrations of molecules. Single molecule SERS was first observed in 1997 through the work of Nie and Emery [26]. This phenomenon occurs when plasmonic nanostructures are brought within a certain distance of each other resulting in the creation of 'hot spots' (first studied by Stockman et al. [25] in 1996), which are areas of intense electromagnetic field strength - leading to extremely large SERS enhancement factors, in the case of
Rhodamine $6 \mathrm{G}$ of the order of up to $10^{14}-10^{15}[26]$. These marked enhancements are of great interest as it makes the detection of ultra low concentrations of molecules (perhaps even single molecules) of biomedical and other interests possible.

Ebbessen et al. in 1998 [27] demonstrated 'extraordinary optical transmission through sub-wavelength hole arrays'. This was due to optical coupling of incident light with plasmons from sub-wavelength hole arrays in $\mathrm{Ag}$ films. This use of SPPs is particularly promising for optical waveguides.

Other more recent milestones in the plasma physics field include the discovery of carbon nanotubes in an arc discharge in 1991 by Ijima - this is a notable linkage between plasma physics and nanoscience. This increasing convergence of the plasma physics field with the emerging micro- (and then nano-) technology disciplines led to the formation of the Plasma Nanoscience research field, the main principles of which were formulated fairly recently and will be discussed in greater detail in Section 4 .

\section{Plasmons for Plasma physics: the physics of surface waves}

\subsection{General characteristics of surface wave discharges}

As noted in the introduction, surface wave plasma (SWP) resonance sustained discharges [49] have been investigated 
for a number of decades, first as a promising diagnostic tool (e.g., elemental and spectral analysis [49]) and more recently as high-density, large-area plasma sources [46]. The use of surface waves in large-area processing has seen them move from an object of purely academic research to a viable fabrication route. More interesting is the idea that the physics of surface waves (SW) is easily extended to the physics of surface plasmons. Moreover, these classical expressions can be fairly easily extended to deal with phenomena that can be regarded as quantum in nature. This will be discussed in more detail in Section 3.3.

Suffice to say, for our purposes the main things we need to know in general about SWPs is that [15]:

- The discharge can be sustained far away from the launcher.

- A broad range of excitation frequencies may be used (i.e., from $10 \mathrm{MHz}$ to $10 \mathrm{GHz}$ - however, the most common frequencies used are $2.45 \mathrm{GHz}$ or $915 \mathrm{MHz}$ ).

On that note, it should be mentioned that the choice of the frequency of the power source is determined by the desired plasma density (to meet the resonance frequency in any particular geometry and reactor size) which in turn is controlled by the pressure, feedstock gas and amount of power we can couple to the discharge. Since, typically a dense plasma of $\geq 10^{11} \mathrm{~cm}^{-3}$ is sought after (for semiconductor production the plasma must be dense), then a matching $\mathrm{GHz}$ excitation frequency should be used. Similarly, in plasmonics, it is the available number density of electrons in a low-loss metal that determines the choice of the frequency of the EM radiation (for example, the wavelength of a laser). These parallels will be discussed in greater detail in Section 3.3.

Other salient features of SWPs include [15]:

- The gas pressure range used is quite large - i.e., from sub-mTorr under electron cyclotron resonance (ECR), to a few atmospheres. Subsequently, there is a large range for the plasma density [15].

- Given the recent design modifications, use of SWPs is quite flexible and energy efficient for a variety of applications.

- SWPs are extensively modelled [15,50-54] (and hence well understood).

- Regarding the surface wave modes that sustain SW discharges, the notation is as follows $\mathrm{TM}_{m n s}$, where $m, n$ and $s$ are the azimuthal, radial and axial indices, respectively and TM refers to a transverse magnetic wave (similarly TE is a transverse electrical wave) [55].

In addition to thinking of these plasmas as linear surface wave discharges or planar surface wave discharges, we can also categorize SWs as propagating (see Sect. 2.2) or standing (see Sect. 2.3) and the plasmas as plasmas produced by the propagating and standing surface waves, respectively.

\subsection{Plasmas sustained by propagating surface waves}

As noted in Section 1.2, a surface wave sustained discharge $[56,57]$ is a plasma generated by evanescently de- caying EM surface waves propagating along a discharge tube $[58,59]$, an example is shown in Figure 4a. A typical set-up is shown in Figure 4b $[45,46]$. Figure 4 b shows a standard linear/column set up, with a SW launcher (i.e., surfatron/surfaguide) and a dielectric tube which the plasma is contained in. Such plasmas are of limited volume and are not uniform - a clear disadvantage for materials processing [45]. Simple tweaks are possible to improve performance, i.e., axial non-uniformity can be avoided by placing a wave launcher at each end of plasma tube, which causes the wave to be reflected at the end of the tube [15] (this can also result in a standing SWP [60]).

The dispersion relation for a TM propagating surface wave is [61]:

$$
\omega^{2}=\omega_{p}^{2} / 2+c^{2} k^{2}-\left(\omega_{p}^{2} / 4+c^{4} k^{4}\right)^{1 / 2},
$$

where $c$ is the speed of light and $k$ is the wavenumber.

Essentially these SWs propagating along a dielectric tube are macroscopic microwave analogues of the surface plasmon polaritons discussed in Section 1.2.

\subsection{Plasmas sustained by standing surface waves}

Figure 4c shows a planar surface wave plasma source [62], where instead of a dielectric tube, the plasma is sustained below a dielectric plate. There are numerous variations on these planar designs, leading to improved densities, homogeneity, etc. We refer the interested reader to a number of articles for a thorough examination of the various source configurations $[15,45,50,55,63-68]$. In this case, it is the plasma sustained by standing SWs that is of interest.

The dispersion equation for a standing transverse magnetic surface mode $\left(\mathrm{TM}_{m n s}\right)$ is [64]:

$$
\left(\gamma_{d} / \varepsilon_{d}\right) \tanh \left(\gamma_{d} d_{t h}\right)=\gamma_{p} / \varepsilon_{p}
$$

where $\gamma_{d}$ and $\gamma_{p}$ are the inverses of the penetration depths (where $\gamma=-\left(\kappa^{2}-\varepsilon \omega^{2} / c^{2}\right)^{1 / 2}[64]$ ) in a dielectric window and plasma, respectively and $d_{t h}$ is the thickness of the dielectric layer.

The frequency of resonance may be obtained from [55]:

$$
\frac{\omega_{p}^{2}}{\omega^{2}}=1 \pm\left(\sqrt{\frac{\omega^{4}}{4 c^{2} \kappa^{2}}+\frac{\beta_{d}}{\varepsilon_{d p} \kappa^{2}} \tan ^{2}\left(\beta_{d} d_{d p}\right)}-\frac{\omega^{2}}{2 c^{2} \kappa^{2}}\right)^{-1}
$$

where $\varepsilon_{d p}$ and $d_{d p}$ are the permittivity and thickness of the dielectric plate, the transverse wave number is $\kappa=U_{m n} / R$ where $U_{m n}$ is the $n$th root of the $m$ order Bessel function and $R$ is the chamber radius, whereas the axial wavenumber is $\beta_{d}=\sqrt{\varepsilon_{d p} \omega^{2} / c^{2}-\kappa^{2}}$. For pure surface modes, $s=0$. Essentially these $\mathrm{TM}_{m n s}$ (via a large dielectric plate) are big microwave analogues of localised surface plasmons from the optical range. Examples of discrete, pure surface modes [46] in the microwave frequency range of our interest are: $\mathrm{TM}_{53}, \mathrm{TM}_{62}$, and $\mathrm{TM}_{33}$.

For more details about current efforts in modelling a range of SW discharges (both planar and tubular) we refer the interested reader elsewhere [69]. Further relevant 
Table 1. Relevant equations and parameters for comparison of surface wave plasmons and surface wave plasmas. Symbols are defined as in the main text, Table 2 or as follows: $n_{c}$ is carrier concentration and $w$ is the width of a graphene micro-ribbon [143].

\begin{tabular}{|c|c|c|c|c|c|c|}
\hline No. & Name & $\lambda$ or freq. & Dispersion equation & $n_{e}\left[\mathrm{~cm}^{-3}\right]$ & Freq. of resonance & References \\
\hline 1 & $\begin{array}{l}\text { SW TM } \\
\text { mode } \\
\text { (travelling) }\end{array}$ & $\mathrm{MHz}-\mathrm{MW}$ & $\begin{array}{l}\omega^{2}=\omega_{p}^{2} / 2+c^{2} k^{2} \\
-\left(\omega_{p}^{2} / 4+c^{4} k^{4}\right)^{1 / 2}\end{array}$ & $\geq 10^{11}$ & $\omega_{S W}=\omega_{p} / \sqrt{1+\varepsilon_{d}}$ & $\begin{array}{c}\text { equation }(12), \\
{[61]}\end{array}$ \\
\hline 2 & $\begin{array}{l}\text { SW TM } \\
\text { mode } \\
\text { (standing) }\end{array}$ & MW & $\begin{array}{c}\left(\gamma_{d} / \varepsilon_{d}\right) \tanh \left(\gamma_{d} d_{t h}\right)= \\
\gamma_{p} / \varepsilon_{p}\end{array}$ & $10^{11}-10^{12}$ & equation (14) & {$[55,64]$} \\
\hline 3 & Bulk plasmon & $\begin{array}{l}\text { NIR, Vis, } \\
\text { UV }\end{array}$ & $\omega^{2}=\omega_{p}^{2}+c^{2} k^{2}($ trans $)$ & $10^{22}$ & $\omega_{p}$ & {$[9]$} \\
\hline 4 & SPPs & $\begin{array}{l}\text { NIR, Vis, } \\
\text { UV }\end{array}$ & $\begin{array}{l}\omega^{2}=\omega_{p}^{2} / 2+c^{2} k^{2} \\
-\left(\omega_{p}^{2} / 4+c^{4} k^{4}\right)^{1 / 2}\end{array}$ & $10^{22}$ & $\omega_{p} / \sqrt{2}$ & {$[219]$} \\
\hline 5 & LSPs & $\begin{array}{l}\text { NIR, Vis, } \\
\text { UV }\end{array}$ & geometry dependent & $\begin{array}{c}10^{22}-10^{23}(\mathrm{Au}, \\
\mathrm{Ag}, \mathrm{Cu}, \text { etc. })\end{array}$ & $\begin{array}{c}\text { when } \varepsilon_{r}(\omega) \approx \\
-2 \varepsilon_{\text {med }}(\text { metal })\end{array}$ & {$[78]$} \\
\hline 6 & $\begin{array}{l}\text { Plasmons- } \\
\text { graphene }\end{array}$ & THz, NIR & $\begin{array}{c}\omega^{2}=\omega_{2 D}^{2}\left(1+e^{2 \mid \bar{q} z_{0}}\right. \\
\left.\left(\omega_{p}^{2} /\left(2 \omega^{2}-\omega_{p}^{2}\right)\right)\right)\end{array}$ & $10^{16}-10^{19}$ & $\omega_{p} \propto n_{c}^{1 / 4} w^{-1 / 2}$ & {$[143-147,164,220,221]$} \\
\hline
\end{tabular}

equations and parameters for SWPs including the resonant frequency, dispersion relation etc. are listed in Table 1 in Section 3.3.

Current research: Recent developments in antenna design have led to the production of a $1.3 \times 1.1 \mathrm{~m}^{2}$ surface wave sustained plasma at $915 \mathrm{MHz}$, with uniform plasma of $\sim 2.7 \times 10^{11} \mathrm{~cm}^{-3}$ (electron number density, standard deviation $\sim 5 \%$, operating pressure $10 \mathrm{~Pa}$ ) [66]. Such large-area plasmas are of great promise for the microelectronics and photovoltaics industries, in particular for the production of large liquid crystal displays and solar cells. Plasmas, in general, have been used to process Si panels approaching $4 \mathrm{~m}^{2}$ [70].

\section{Plasmons for photonics: the physics of surface plasmons}

\subsection{Physical foundations for surface plasmons}

The physical foundations for surface plasmons consist of a few major milestones including, but not limited to: Mie theory [19] for absorption and scattering of light by spherical particles and the Drude free electron model for metals etc. [18]. The Drude model assuming free electrons in a metal is given by [71]:

$$
\varepsilon(\omega)=1-\frac{\omega_{p}^{2}}{\omega^{2}},
$$

where $\omega_{p}$ is the plasma frequency, defined in equation (2). It should be noted that Mie theory, by itself, is geometricthe effect of a plasma is taken into account when the Drude model is used to describe the dielectric function of the metal (see Sect. 4.4 for an expanded discussion).
The extinction and scattering cross sections arising from Mie's solutions to Maxwell's equations (when the particle is much less than wavelength, $\lambda$ ) are [72]:

$$
\begin{aligned}
\sigma_{e x t} & =\frac{18 \pi \varepsilon_{m e d}^{3 / 2} V}{\lambda} \frac{\varepsilon_{i}(\lambda)}{\left[\varepsilon_{r}(\lambda)+2 \varepsilon_{m e d}\right]^{2}+\varepsilon_{i}(\lambda)^{2}}, \\
\sigma_{\text {scat }} & =\frac{32 \pi^{4} \varepsilon_{m e d}^{2} V^{2}}{\lambda^{4}} \frac{\left(\varepsilon_{r}-\varepsilon_{m e d}\right)^{2}+\left(\varepsilon_{i}\right)^{2}}{\left(\varepsilon_{r}+2 \varepsilon_{m e d}\right)^{2}+\left(\varepsilon_{i}\right)^{2}},
\end{aligned}
$$

where $V$ is the volume of the particle, $\tilde{\varepsilon}=\varepsilon_{r}+i \varepsilon_{i}$ is the complex metal dielectric function, where $\varepsilon_{r}=n_{r}^{2}-n_{i}^{2}$ and $\varepsilon_{i}=2 n_{r} n_{i}$ are the real and imaginary parts of $\tilde{\varepsilon}$ and $n_{r}$ and $n_{i}$ are the real and imaginary parts of the complex refractive index of the metal, and $\varepsilon_{m e d}=n_{m}^{2}$ is the dielectric function of the medium [72].

Generalisations of these equations for spherical particles of any other aspect ratio is possible via Rayleigh-Gans theory $[9,73,74]$ (for a more detailed coverage, please refer to Bohren and Huffman [9]). For other shapes, however, analytical solutions are not available [72] and they must be studied numerically or via plasmon hybridization theory. The advent of plasmon hybridisation theory [75] in 2003 has made it easier to analytically describe plasmon modes in a range of exotic nanostructures, from nanoshells [75], to nanorice [76], to nanotubes with dielectric cores [77]. This is done by describing the interaction of plasmon modes supported by basic structures. For example, plasmon modes of nanoshells could be described as two fundamental dipolar modes [11], with one mode supported on the outside of a larger outer nanosphere and one mode on the surface of a smaller inner dielectric nanosphere or 'void' [77]. Some further important relations, i.e., dispersion equations and resonant frequencies of SPPs and LSPs are listed in Table 1 and discussed in greater detail in Section 3.3. 
Table 2. List of symbols and abbreviations from manuscript and their meanings, the equation and/or first section they are mentioned in.

\begin{tabular}{|c|c|c|}
\hline Symbol & Meaning & Eq. or Section \\
\hline$\omega_{p}$ & Plasma frequency & 2 \\
\hline$E_{\text {bulk }}$ & $\begin{array}{c}\text { Energy of a bulk/volume } \\
\text { plasmon }\end{array}$ & 1 \\
\hline SPP & Surface Plasmon Polariton & Section 1.2 \\
\hline$E_{S P P}$ & $\begin{array}{c}\text { Energy of a surface plasmon } \\
\text { polariton }\end{array}$ & 6 \\
\hline$\omega_{s p}$ & SPP frequency & 7 \\
\hline LSP & Local Surface Plasmons & Section 1.2 \\
\hline$E_{L S P}$ & Energy of a local surface plasmon & 8 \\
\hline$n_{e}$ & the electron density & 2 \\
\hline$e$ & the charge of an electron & 2 \\
\hline$m_{e}$ & the rest mass of an electron & 2 \\
\hline$\overline{v_{p h}}$ & $\begin{array}{c}\text { phase velocity of transverse bulk } \\
\text { plasmon }\end{array}$ & 4 \\
\hline$v_{g}$ & $\begin{array}{c}\text { group velocity of transverse bulk } \\
\text { plasmon }\end{array}$ & 5 \\
\hline$c$ & speed of light & 5 \\
\hline$\lambda$ & wavelength & 16,17 \\
\hline$k$ & wavenumber & 5 \\
\hline$\tilde{\varepsilon}$ & $\begin{array}{c}\text { complex metal dielectric } \\
\text { function }\end{array}$ & 16,17 \\
\hline$\varepsilon_{i}$ & imaginary part of $\tilde{\varepsilon}$ & 16,17 \\
\hline$\varepsilon_{r}$ & real part of $\tilde{\varepsilon}$ & 16,17 \\
\hline$\varepsilon_{\text {med }}$ & dielectric function of the medium & 16,17 \\
\hline$\sigma_{e x t}$ & extinction cross section & 16 \\
\hline$\sigma_{\text {scat }}$ & scattering cross section & 17 \\
\hline$V$ & volume of the nanoparticle & 16,17 \\
\hline$n$ & refractive index & Section 3 \\
\hline$Q_{L S P R}$ & $\begin{array}{l}\text { quality factor of plasmonic } \\
\text { material for LSPR-spherical }\end{array}$ & 19 \\
\hline$Q_{L S P R}^{\prime}$ & $\begin{array}{c}\text { quality factor of plasmonic } \\
\text { material for LSPR-ellipsoidal }\end{array}$ & 19 \\
\hline$E(\lambda)$ & LSPR extinction & 18 \\
\hline$\chi$ & $\begin{array}{c}\text { form factor for nanoparticle } \\
\text { aspect ratio }\end{array}$ & 18 \\
\hline$\theta_{K}$ & Kretschmann angle & 10 \\
\hline$\varepsilon_{1}$ & $\begin{array}{c}\text { permittivity of a glass hemisphere, } \\
\text { for excitation of SPPs in Kretschmann } \\
\text { configuration }\end{array}$ & 10 \\
\hline$\overline{\varepsilon_{m}}$ & $\begin{array}{l}\text { permittivity of metal film in } \\
\text { Kretschmann configuration }\end{array}$ & 10 \\
\hline$\varepsilon_{2}$ & $\begin{array}{l}\text { permittivity of medium in upper } \\
\text { half plane above metal film in } \\
\text { Kretschmann configuration }\end{array}$ & 10 \\
\hline$\Delta \lambda_{\max }$ & $\begin{array}{c}\text { Maximum } \lambda \text { shift on adsorption } \\
\text { of a biospecies }\end{array}$ & 21 \\
\hline$n_{b u l k}$ & $\begin{array}{c}\text { bulk refractive index of } \\
\text { nanoparticle }\end{array}$ & 21 \\
\hline$\Delta n$ & Change in $n$ caused by adsorbate & 21 \\
\hline$d$ & adsorbate layer thickness & 21 \\
\hline$l_{d}$ & EM field decay length & 21 \\
\hline SERS & $\begin{array}{c}\text { Surface Enhanced Raman } \\
\text { Scattering } \\
\end{array}$ & Section 1.3 \\
\hline$E F_{S E R S}$ & SERS enhancement factor & 11 \\
\hline$I_{S E R S}$ & Intensity of SERS signal & 11 \\
\hline$I_{N R S}$ & Intensity of Raman signal & 11 \\
\hline$N_{\text {surf }}$ & $\begin{array}{l}\text { number of molecules bound to } \\
\text { SERS substrate }\end{array}$ & 11 \\
\hline$N_{v o l}$ & $\begin{array}{l}\text { number of molecules in the } \\
\text { excitation volume }\end{array}$ & 11 \\
\hline
\end{tabular}

Quickly revisiting equations (16) and (17), for a single nanoparticle (NP), Mie theory has the extinction (sum of absorption and scattering cross sections) as [78]:

$$
E(\lambda) \propto\left[\frac{\varepsilon_{i}}{\left(\varepsilon_{r}+\chi \varepsilon_{m e d}\right)^{2}+\varepsilon_{i}}\right],
$$

where $\chi$ is a form factor related to the nanoparticle's aspect ratio (2 for a sphere) [78]. Local surface plasmon resonance occurs at the maximum of the right hand side of equation (18), which is where $\varepsilon_{r}(\omega) \approx-2 \varepsilon_{\text {med }}$ (when $\left.\left|\varepsilon_{r}\right| \gg \varepsilon_{i}\right)$ [78]. It should be noted that classical Mie theory (unmodified) is not appropriate for interacting nanostructures (this is particularly important to note when designing arrays intended to take advantage of plasmonic effects).

\subsection{Materials design for surface plasmons}

When thinking about what type of nanostructure to grow, one must have a very specific application in mind. This is because the size, shape, composition, spacing etc will affect how the nanostructure reacts. The number of free carriers determines the performance of the plasmonic material. For example, something as simple as composition a $633 \mathrm{~nm}$ laser will excite Au whereas $532 \mathrm{~nm}$ is more appropriate for Ag. What also must be considered - is the price of the material, worth the application? For example, is a better plasmonic quality factor worth extra cost? Note that the plasmonic quality factor varies for different geometries and for metals it is [79]:

$$
Q_{L S P R}(\omega)=-\frac{\varepsilon^{\prime}(\omega)}{\varepsilon(\omega)}, Q_{L S P R}^{\prime}(\omega)=\frac{\varepsilon^{\prime}(\omega)^{2}}{\varepsilon^{\prime \prime}(\omega)},
$$

where $Q_{L S P R}$ is for spheres and $Q_{L S P R}^{\prime}$ is for ellipsoids [79].

Sodium and Potassium have higher quality factors but they are so volatile that they cannot be practically used by themselves (imagine if Potassium came into contact with water - then consider that a lot of the biospecies that have to be analysed using LSPR may be in aqueous solution.). Silver, for example has a higher $Q$ than $\mathrm{Au}$, however it is toxic - so therefore not appropriate for some bio-related applications [80]. Clearly there are a lot of factors to consider - having the best performing material is not always the way to go, it is more like having the best fit material for a number of considerations both performance (high quality factor) and practicality (ease of fabrication, excitation in the visible range of the EM spectrum, i.e., white light), in other words - the choice of material is a balancing act. The optical properties of $\mathrm{Ag}$ and $\mathrm{Au}$ in the visible range can be described via $\tilde{\varepsilon}$, the complex metal dielectric constant [78]. Nevertheless, the electron density, which determines the frequency of plasma resonance still remains the main factor in the material choice.

Whilst the 'coinage' metals ( $\mathrm{Au}$ and $\mathrm{Ag}$ ) possess acceptable plasmonic merit, the reason they are more widely used than other materials which may have a greater number of free carriers (i.e., Alkali metals such as $\mathrm{Na}$ and $\mathrm{K}$ ) 
is due to their relative inertness and experimental convenience (i.e. they are more convenient than the Alkali metals whose volatility makes them difficult to use) [81]. In fact it is well recognised that both of these materials are quite lossy, that is the signal carried on these materials degrades very rapidly (not a huge issue for sensing, but a big problem for application in an 'all optical chip'). This has led researchers to consider using alternative, combinatorial materials such as doped metals, alloys or intermetallic compounds, all of which enable a greater degree of flexibility when attempting to tune the plasmon resonance [81-86] which is one of the drawbacks in just using pure $\mathrm{Au}$ or Ag. As yet, the barrier to widespread implementation of these intermetallic compounds or alloys for plasmonic applications is the lack of carefully designed, commercially viable fabrication methods with a high degree of control over size, shape, composition and placement of plasmonic nanoparticles.

Hence a checklist to consider when choosing the material to use for plasmonic nanostructures is:

- Does it have a matching electron density?

- Does it have low losses?

- Does it have an acceptable Quality factor?

- Is it convenient to use/produce?

- How to fabricate it, including nanoarrays?

- Is it robust/relatively inert?

- Can it be integrated into existing technology?

- Does it work in the visible range of the EM spectrum?

- Is it comparatively cheap?

Surface plasmons are very sensitive to their local surroundings, leading to a wavelength shift, a change in angular momentum or a change in intensity if there is a modification in the local refractive index. These characteristics are detectable and may be used to great effect in sensors. As well as sensors, LSPs are useful for solar cells, optical computing and biomedical applications (including cancer treatment) [87].

For smaller nanostructures (radius $<\sim 30 \mathrm{~nm}$ ), absorption dominates LSPR extinction, whereas scattering dominates for larger nanostructures (radius $>\sim$ $30 \mathrm{~nm}$ ) [80] - consider equations (16) and (17) (this is one of the reasons that larger Ag nanostructures are promising for plasmon enhanced solar cells (and LEDs) - the scattering is enhanced leading to an enhancement in the solar cell efficiency [88-90]). Regarding shape [91,92] - it is documented that there is higher refractive index sensitivity for higher aspect ratios i.e., $157-497 \mathrm{~nm} / \mathrm{RIU}$ from aspect ratio of 1.0 (sphere) to 3.4 (nanorods, described by spheroidal model) [72], where RIU is refractive index unit. Moreover, nanostructures featuring sharper tips also exhibit enhanced sensitivity to changes in their immediate surrounding environment [72]. This may also be linked to hot spot enhancements around the tips of nanostructures due to the so-called "lightning rod effect" [11]. For the cases where nanostructures are non-spherical, the number of resonances increases as the facets increase, e.g., a cube has more plasmon resonances than a triangle, which has more than a sphere [80]. In other words, if the structure is 'anisotropic' it can support multiple plasmon modes, the number of which depends on the number of ways the shape can be polarised $[78,80]$. For example, for metallic nanorods, two plasmon modes are present, namely a longitudinal mode (polarisation parallel to the long axis, red shifted) and a transverse mode (polarisation perpendicular to the long axis, blue shifted) [78]. Here we stress that quite similar polarisation effect happen in (gaseous) plasma waveguides which determine their eigenmodes [56].

As well as size and shape, tailoring the spacing between nanostructures is another way to control the plasmonic response of the array. For example, Jain et al. [93] derived the 'plasmon ruler' equation as:

$$
\frac{\Delta \lambda}{\lambda_{0}} \approx 0.18 \exp \left(\frac{-s / D}{0.23}\right)
$$

where $\Delta \lambda / \lambda_{0}$ is the observed plasmon shift, $s$ is the interparticle edge-edge separation and $D$ is the particle diameter - for a pair of $\mathrm{Au}$ particles in a protein medium. Depending on how close the nanostructures are to each other, their surface plasmons may constructively interfere and result in a marked enhancement in the local electric field. This is particularly important in the case of 'hot spot' generation. Hot spots [25] are the term given to areas of intense electric field enhancement [41,94-96]. These areas are particularly useful for single molecule sensing (and can often lead to SERS enhancements of up to $10^{14}$ ). However, controlling (or even predicting) where hot spots will occur is very challenging. This means particular attention (and thorough modelling efforts) need to be directed at understanding and finding fabrication methods capable of controlling the coupling between plasmonic nanostructures with various sizes, shapes, composition, spacing and orientations [91,97-107]. The tailoring of NP size, shapes and array properties such as ordering, regularity etc. is what is commonly pursued in Plasma Nanoscience and will be discussed in greater detail in Section 4 .

\subsection{Comparison of plasmas and plasmons}

Let us now summarise the collective phenomena discussed in Sections 2 and 3, through comparison of their main equations and features as presented in Table 1.

Just looking at the energies of the oscillations confirms the common ground - i.e. compare the energy derived from equation (9): $E=\hbar \omega_{p} / \sqrt{2}$, which is identical to $E_{S P P}$ (Eq. (6)) and only differs by a factor of $1 / \sqrt{2}$ and $\sqrt{3} / \sqrt{2}$ for $E_{\text {bulk }}$ (Eq. (1)) and $E_{L S P}$ (Eq. (8)). However, it is not just an issue of energies and resonant frequencies, the synergism of the plasma physical phenomena and plasmonic physical phenomena follows from this table.

For example, compare the dispersions relations of surface plasmons (Row 4) and TM surface plasma modes travelling (Rows 1 ) - the form is exactly the same in gas plasma and metal plasma (the dispersion relation in (1) is the same as the dispersion relation in (4), despite there being about a $10^{11} \mathrm{~cm}^{-3}$ difference in electron number density. In particular, as noted in Sections 2.2-2.3 the TM 
modes in a SWP via a dielectric tube and a SWP via a dielectric plate are analogous to surface plasmon polaritons and localised surface plasmons, respectively.

\section{Everything old is new again: plasma nanoscience meets plasmonics}

\subsection{Introduction to plasma nanoscience}

Plasma nanoscience [108-112] is a research field which incorporates elements of plasma physics, nanoscience, materials science and engineering, physical chemistry and surface science, that is centered on elucidating how a plasma-based growth environment may be used to bring self organisation of nanostructures up to the as yet elusive, deterministic level $[6,7,34,110,113,114]$. A deterministic fabrication process is one where it can be predicted with a great degree of certainty what type of nanostructure will be produced, from what type and how material is deposited on a surface. This idea of determinism is particularly important for the fabrication of nanoarrays for plasmonic applications where control of size, shape, elemental composition and positioning is crucial. Finding a growth/processing environment that is capable of precisely 'tuning' these parameters is a significant challenge. Low-temperature, non-equilibrium plasmas are a particularly versatile tool for this purpose [6] and may be used for all stages of the growth process from generation of building and working units in the plasma bulk (building units are the material that make up the nanostructure, working units prepare the surface for deposition), through to surface preparation, directed transport of materials to the surface, nanoassembly of highly-tailored structures as well as functionalisation and post-processing of arrays. A review by Anders [115] highlighted the use of plasmas of metal vapours for production of metal nanostructures. An interesting point to note is that these plasmas of metal vapours can be tailored to produce metal plasmas (i.e., collective electron oscillations with respect to the positive ion background in the metal nanoparticles from the condensing of the metal vapour).

As noted previously [113], a range of factors need to be considered when choosing a fabrication technique, some of these factors include:

- Process control.

- Energy efficiency of the fabrication process.

- Quality of the material output.

- Integration with existing technology.

- High throughput.

- Large area and volume processing.

- Simplicity - i.e. is it easy to use?

- Cost.

- Safety factors - i.e., is it human health benign?

- Environmental impact.

In reference to this checklist-style approach, there are a number of reasons for choosing a plasma as a nanofabrication environment over other techniques. Firstly, plasmabased systems offer a number of effective process control 'knobs' such as gas inlet, pressure, partial pressure of the gases, applied power, applied voltage, degree of ionisation, etc. These knobs can be manipulated, resulting in an efficient growth process. Secondly, in order for plasmonic devices to be widely adopted, they must be able to be easily integrated into existing semiconductor-based devices. Wet chemical production methods are not easily integrated into existing manufacturing lines. In contrast, over $50 \%$ of semiconductor manufacturing lines are plasma-based [116]. This means that by using plasmas as a fabrication basis, existing production lines could be used to make plasmonic nanostructures that could be easily integrated into current devices, improving their performance and efficiency (both in terms of energy and matter [108]), without the need for a costly, full-scale overhaul of production lines. Moreover, a plasma-based fabrication method is capable of large-area (and large volume) processing as noted above $1.3 \times 1.1 \mathrm{~m}^{2}$ surface wave plasmas have recently been achieved [66].

\subsection{Tailoring metal nanoarrays}

In order to optimize the sensitivity and performance of plasmonic based devices, it is necessary to precisely tailor the nanoarray parameters as noted in Section 3. The most significant parameters to control include: nanostructure size, shape and composition as well as nanoarray spacing and surface coverages.

For the majority of modern technological devices, a regular array of uniform components is required [117]. This is because the coupling between nanostructures in the array must be able to be controlled to tailor the optoelectronic properties of the device. However, there are a few obstacles in obtaining size-uniform nanostructures with high throughput and at a low cost. Plasmabased growth methods [115,118-120] have been shown to be promising controllable bottom-up alternatives for size-uniform and size-controlled growth. For example, it was demonstrated numerically $[118,119]$ that plasmas lead to a greater degree of nanostructure size uniformity than is possible in a neutral gas-based selforganized process. Highly-ordered nanostructure arrays have been reported (experimentally) using inductively coupled plasmas (ICPs) [121], atmospheric microwave plasma torches [122], single-step deep-reactive-ion etching [123] and a range of other plasma-based techniques. This is particularly important for nanoplasmonics as controlling the coupling between nanostructures by varying size and position is a way to fabricate devices which can take advantage of plasmonic effects.

Regarding controlled shapes, plasma-based growth methods have been used to grow a large variety of nanostructures - from quantum dots, nanotips, graphene, even nanoarchitectures (i.e., Ag islands connected by carbon nanowires - the first steps to self-organised nanocircuitry growth [120]). Controlling nanostructure shape is particularly important in plasmonic applications, as mentioned in Section 3. For example, it has been demonstrated that sharper tips and higher aspect ratios lead to the increased 
sensitivity to changes in the refractive index [72]. Indeed, plasma effects (i.e. resonance around the plasma frequency) will affect intra- and interparticle coupling depending on the nanoparticle size, shape, and distance between them. Recall from Sections 3.1 and 3.2, the LSP properties are determined by the plasma density and 3 geometric factors (see Eqs. (16) and (17) for the influence of size, Eq. (18) shape and Eq. (20) for distance).

Low-temperature plasmas have been demonstrated to produce sharper carbon nanotips than is possible in a neutral gas process [124]. Moreover a recent review on the use of metal vapour plasmas for the growth of metal nanostructures [115], noted that there were a number of options including ionised physical vapour deposition (iPVD) based on sputtering (more appropriate for Ag), as well as pulsed laser deposition, filtered cathodic arcs, etc. The benefit of using plasma-enhanced magnetron sputtering is that it is possible to exert a high level of control over both the direction and the energy of the charged particles. Indeed, both those factors are responsible for dislodging the metal atoms from the target, as well as ionising and then controlling the metal atoms themselves. This in turn affects the way particles self-organise into a nano-array. Whilst the most popular methods to form nanostructures for plasmonics are currently e-beam and nanosphere lithography and nanotemplating, plasmas are a useful alternative that can be used to control the deposition of metals or metal oxides through nanoporous templates to create size uniform, regular arrays [125,126], as well as in conjunction with nanosphere lithography [127]. This control over the generation of nanostructure material, transport to and interaction with the surface is enabled by careful modification of the plasma parameters such as power, pressure, gas composition, etc. as well as the external surface bias [128-132].

Changing nanostructure size and shape, however, can be problematic in certain biological and nanoelectronic applications [116,133]. As mentioned in Section 3, an alternative is to change elemental composition and internal structure (i.e., core/shell layers, compositionally graded structures, etc.). This enables another possibility, namely the composition control. Using more complex materials (i.e., binary and ternary alloys, etc.), however, brings the issue of control back. The growth process must be tailored so that materials may be grown not only with the desired shape and size, but also the right composition and internal structure. This necessitates thorough modelling efforts to determine exactly what fabrication method will result in structures with the size, composition, shape and internal structure that will in turn generate the desired plasmonic response. Gaseous plasmas represent an ideal fabrication environment for this purpose. A high degree of compositional control during nanoassembly has been demonstrated for plasma-based processes both computationally [113,116,134,135] and experimentally [136-141].

In addition to manipulating traditional plasmonic materials such as $\mathrm{Au}$ and $\mathrm{Ag}$ by alloying or doping, alternative materials such as graphenes, doped semiconductors, carbon nanotubes [142] and multilayers have also been shown to exhibit plasmonic behaviour. Whilst graphene and its derivatives [143-147] have been used in combination with metallic nanostructures for plasmonic-based sensing [148], it is also useful by itself. In fact, a quasiparticle termed a 'plasmaron' (combination of a plasmon and an electron) was recently observed in free standing graphene [149]. For graphene, plasmons are excited in the near infrared (or $\mathrm{THz}$ range) $[150,151]$. Koppens et al. [152] summarise the main advantage of graphene plasmonics over traditional noble metal-based plasmonics as: tighter confinement, longer propagation distances and high tunability. Moreover, they state that using graphene, may pave the way towards 'quantum plasmonics' [152]. The study of collective quantum effects in plasmas [153-163] and particularly in graphene [164-166] is a significant challenge that is likely to be the subject of intense research efforts in the future. Plasmas have been shown to be a very promising way to make graphenebased structures, the particular benefit is that they may be grown without the presence of a metal catalyst $[148,167]$.

\subsection{Existing and emerging applications}

As shown in Figure 5, there is a range of applications that work based on plasmonic phenomena. Moving clockwise from the top left hand corner, these include: a (proposed) plasmon cloaking device [174,175], single-molecule SERS sensing platforms that rely on plasmonic hot spots [169], theranostics [170], surface plasmon lasers or 'spasers' [173, 176,177], LSPR biosensors using simple LEDs [172], nextgeneration photovoltaic cells [87] and plasmon rulers [171]. This is of course a non-exhaustive list that is meant to just give an idea of how many fields plasmonics is involved in. For the purposes of this section, we will focus mainly on photovoltaic cells, sensors (SERS, SPR and LSPR-based) and biomedical applications.

Photovoltaic cells can utilise plasmons by incorporation of nanoislands/nanostructures into bulk and thin-film Si solar cells. The key is to design the nanostructure geometry such that the enhanced forward scattering into the photoactive layer obtained via coupling of surface plasmons is not overshadowed by strong absorbance of incident light at the plasmon resonance wavelength of the nanoparticles [178]. Recall Section 3, in particular equations (16) and (17) which showed that scattering dominates in larger particles whereas absorbance is more important for smaller particles. By using relatively large $\mathrm{Ag}$ islands (around $50 \mathrm{~nm}$ ) the scattering of light into the $\mathrm{PV}$ cell is enhanced, the plasmon resonance of these particles can be tuned so it is around the desired wavelength by modifying the surrounding local dielectric environment [179]. Note that the enhancement may be optimised by varying the shape, size, material and surface coverage of the nanostructure [178-184]. Figure 6a shows that higher aspect nanostructures (i.e., cylinders rather than spheres) maximises the fraction of incident light scattered into the photoactive layer and hence enhances the solar cell efficiency [180]. Akimov et al. numerically investigated the effect of the the size and surface coverages 


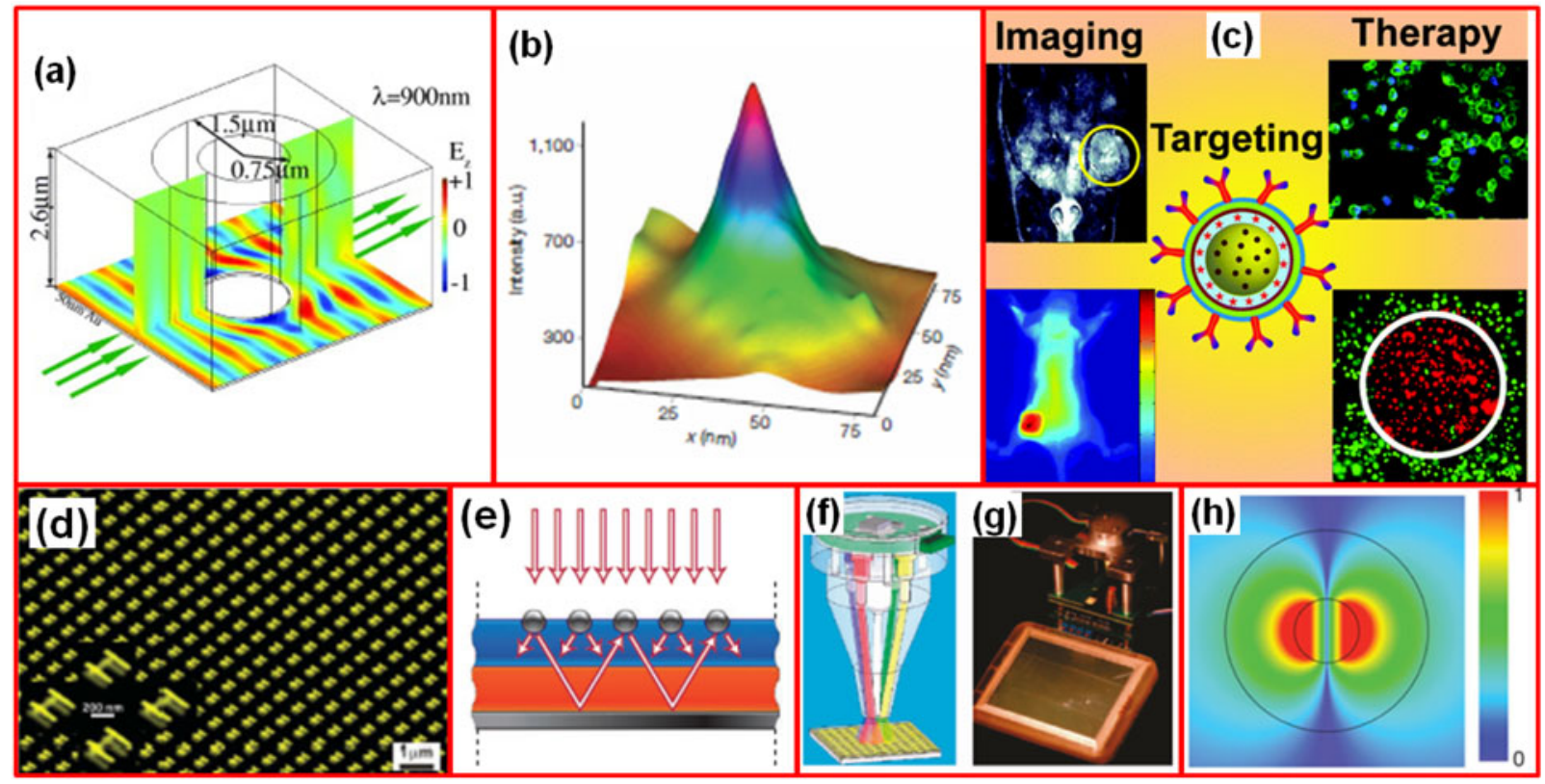

Fig. 5. (a) A plasmon 'cloaking device', reproduced with permission from [168], IOP Publishing Ltd; (b) a plasmonic 'hot spot', reprinted by permission from Macmillan Publishers Ltd: Nature [169], copyright 2011; (c) the various theranostic applications of plasmonic nanoshells, reprinted with permission from [170], copyright (2011) American Chemical Society. (d) Plasmon rulers, from [171], reprinted with permission from AAAS; (e) next generation photovoltaic cells, reprinted by permission from Macmillan Publishers Ltd: Nature Materials [1], copyright 2010; (f) and (g) LSPR biosensors using simple LEDs, reprinted with permission from [172], copyright (2008) American Chemical Society; (h) surface plasmon lasers or 'spasers', reprinted by permission from Macmillan Publishers Ltd: Nature [173], copyright 2009.

of $\mathrm{Ag}$ nanoparticles [183,184] on light absorption in $\mathrm{Si}$ thin film solar cells. They found that for Ag nanoparticles, there were 2 best configurations that would maximise the forward light scattering and minimise absorption by the nanoparticles in the wavelength range of interest, by optimizing the higher order plasmon modes and shifting the lower order resonance modes into a less important wavelength range. This effort was extended to investigate other materials as shown in Figures $6 \mathrm{~b}$ and $6 \mathrm{c}$. In another paper [185] the enhancement due to resonant plasmonic metals $(\mathrm{Ag})$ was separated from the effect of non-resonant plasmonic metals $(\mathrm{Al})$.

Methods to construct nanostructure arrays suitable for this purpose include fragmentation after thermal evaporation [179], as well as plasma-assisted nanosphere lithography [127] and plasma-assisted deposition through nanopore arrays $[125,126]$ discussed in Section 4.2. The benefit there is that the size of the nanoparticles in template-based methods is more controllable than in stress-induced fragmentation [186] which results in a very broad island size distribution. Given the rather large size of the islands required, i.e. $50 \mathrm{~nm}$, plasma-assisted nanosphere lithography and nanopore templates are suitable - in the case where smaller islands are required (often the case for sensor devices), a bottom-up growth route, such as plasma-based self-organisation is more appropriate.

In addition to metal nanoparticles and associated LSPs, metallic films and resultant SPPs may be used to enhance the efficiencies of photovoltaic cells. A good review on various configurations of metallic nanoparticles and metallic films for the purpose of enhancing the efficiencies of solar cells was recently published by Green and Pillai [187]. They note that when metallic films are attached to the rear surface of the photovoltaic cell, the resultant SPPs can significantly enhance photovoltaic cell absorption. Moreover, the combination of a metallic film as a reflector at the back of the cell, combined with sandwiched high index - low index - high index layers and a textured front surface has been shown to significantly enhance light trapping of the photovoltaic cell [187]. They also note that the location of metallic nanostructures (hence resultant LSPs) within or without the cell is important - it has been suggested that the performance of the cell may be improved by placing metallic nanostructures closer to the most active region of the cell, rather than just on the top surface of the cell [187]. It is possible that metallic films, hence SPPs may be used in a similar configuration for this purpose - Green and Pillai include a sketch of a proposed cell based on the work of Wang et al. [188] which consists of a indium tin oxide layer $(55 \mathrm{~nm})$, the rear segment of which contains a $20 \mathrm{~nm}$ layer of $\mathrm{Ag}$ which covers $54 \%$ of the cell; this is followed by a $15 \mathrm{~nm}$ thick amorphous Si layer and then a $50 \mathrm{~nm}$ thick Ag layer as a rear reflector [187].

Sensors based on plasmonics can function optically or electronically. However, here we will focus on SPR, LSPR and SERS-based sensors. Surface Plasmon Resonance 

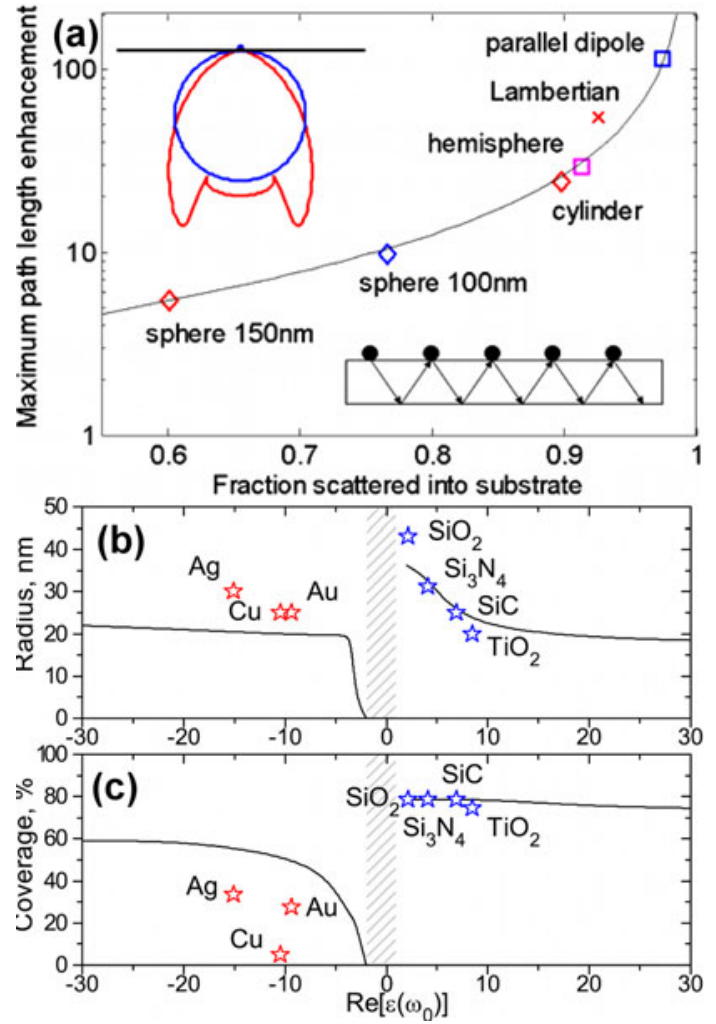

Fig. 6. (a) The effect of nanoparticle shape on the fraction of incident light scattered into the photoactive layer of the solar cell. Reprinted with permission from [180], copyright 2008, American Institute of Physics. The maximum enhancement possible for various materials with (b) radius and (c) surface coverage, where $\operatorname{Re}[\varepsilon(\omega)]$ is the real part of the nanoparticle permittivity. (b) and (c) reprinted with permission from [178], copyright 2010, American Institute of Physics.

sensors work on the basis that an analyte/biomolecule changes the local refractive index in the vicinity of the surface of the metallic film. This in turn changes the resonance of the surface plasmons which can be detected either as a change in angle or change in wavelength of absorption. Sensors working on this principle typically use the Kretschman configuration to excite the surface plasmons (see Fig. 4e). Examples include Wu et al. [189], where a change in biomolecule concentration led to a change in refractive index near the metal surface which leads to change in propagation constant of the SPP which can be measured by attenuated total internal reflection. Commercially available sensors based on SPR such as the BIAcore instrument from Pharmacia have been widely used for a number of decades [190-192]. Another example is a handheld SPR biosensor [193] which could detect changes as low as $3.3 \times 10^{-6}$ refractive index units. Localized surface plasmon resonance (LSPR) [8,194-197] sensors work on a similar principle, although the change measured is wavelength only, not angle. The change in wavelength, $\lambda$ as a result of absorbtion of a biospecies is [8]:

$$
\Delta \lambda_{\max }=n_{\text {bulk }} \Delta n\left[1-\exp \left(-2 d / l_{d}\right)\right]
$$

where the bulk refractive index of the nanoparticle is $n_{\text {bulk }}$, $\Delta n$ is the change in refractive index caused by the adsorbate, $d$ is the adsorbate layer thickness and $l_{d}$ is the EM-field decay length [8]. Neuzil and Reboud [172] constructed a handheld, battery-operated LSPR-based sensor, where 4 light emitting diodes were used in place of a spectrum analyser (a schematic and image of the device are shown in Figs. 5f and $5 \mathrm{~g}$ ). Such a device is particularly promising as a portable, point-of-care detection system - not the least because it is mechanically simpler than an SPR analogue (it is the change in wavelength that is important - not the change in angle of reflection) [172]. Additional refinements to LSPR spectroscopy lie in utilising Fano resonances $[16,40,198]$, the extreme sensitivity to changes in the local environment around the resonant structure suggest that detection of single molecule binding events may be possible [40]. This will likely be an area of intense research efforts in the near future.

Surface enhanced Raman scattering, discussed in Section 1.3, is another popular non-invasive sensing option. A thorough review of SERS is beyond the scope of this colloquium, hence we refer the interested reader to the following articles [95,96,199-202]. Here we will be focussing on SERS relevant structures produced using plasma methods, rather than top-down lithographic approaches. Agarwal et al. [199] fabricated Ag and Au nanoparticle substrates using pulsed laser ablation demonstrating a reasonable degree of control over the bottom-up growth through variation of the chamber pressure and the number of pulses and with a clear SERS response for Rhodamine 6G [199]. Rider et al. [148] recently used a plasma-based approach to grow metal nanoparticles in combination with vertical graphenes to make 3D metal-graphene nanohybrid SERS platforms (see Fig. 7). It was demonstrated that using vertical graphenes, rather than horizontal graphenes, provides a markedly higher effective bookshelf like area where Au nanoparticles can attach. This means that there is a much greater area where analyte species can attach, compared to typical horizontal SERS sensor architectures [148].

Biomedicine and the convergence of plasmonics and plasmas: an example of a biomedical application (excluding sensors) which involves plasmons is photothermal therapy [170,203-205] (see Fig. 5c). The nanoparticle (often $\mathrm{Au}, \mathrm{Au}$-nanoshells and variations) is excited in vivo with light at the resonant frequency of the particle. This serves to excite LSPs, which are then dissipated thermally in the local medium possibly killing the surrounding cells. For example, hollow Au nanoparticles were functionalized with an antibody/ligand that would dock on to an antigen present on the surface of a cancer cell. The area would be exposed to EM radiation that would cause a plasmon to be generated from the NP, which would lead to heat released locally which would selectively kill the cancer cell and leave the surrounding tissue unaffected [206]. It has recently been shown that charged nanoparticles can be less toxic [207], moreover, using plasmas provides a high level of control over the charge and transport of nanoparticles [208]. 

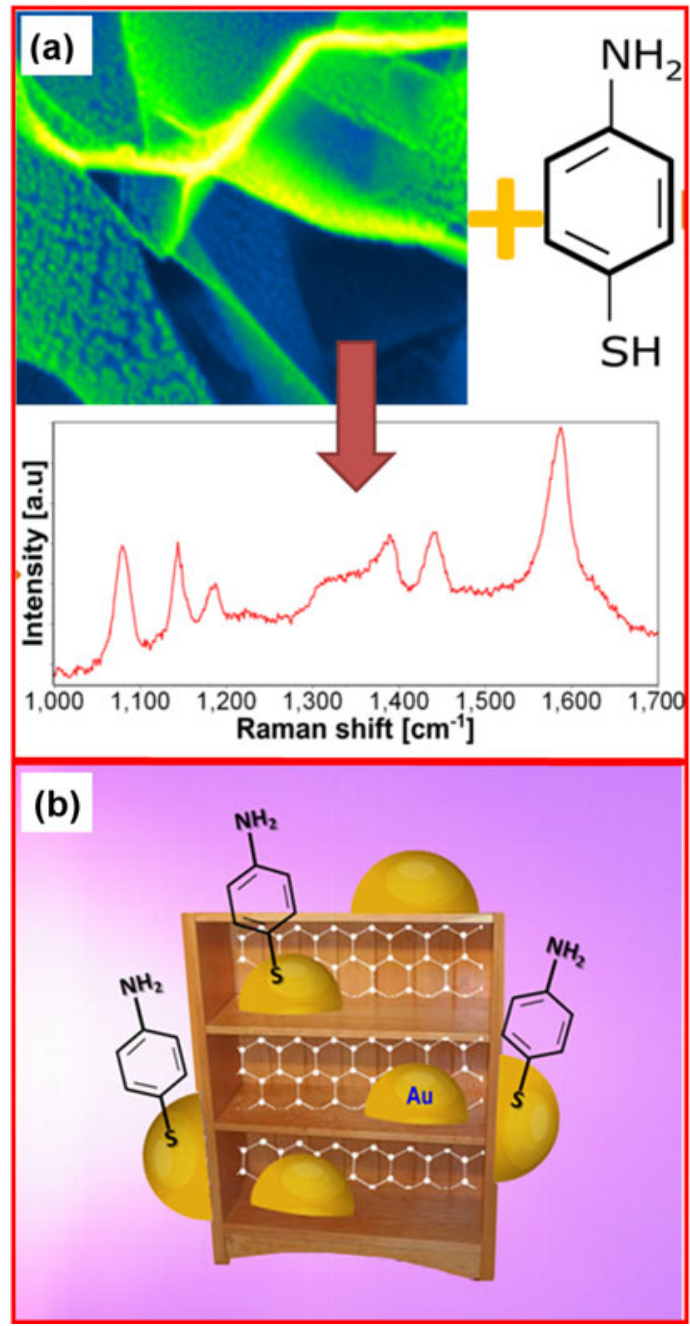

Fig. 7. (a) Au nanoparticle decorated vertical graphenes and a SERS spectra of $10^{-2} \mathrm{M}$ 4-aminothiophenol deposited on $\mathrm{Au}$ decorated vertical graphenes; (b) concept of Au decorated vertical graphenes as a bookshelf-style sensor. (a) and (b) from [148] - reproduced by permission of The Royal Society of Chemistry.

Moving slightly away from nanoplasmonics, there has been a lot of excitement in the last few years centred around the use of atmospheric plasmas in medicine and health care [209]. Specific applications include (but are not limited to): wound treatment, tissue engineering [209], treatment of skin diseases such as Rosacea [210], antimicrobial applications [211], inducing cancer cell apoptosis [212] and minimizing adenovirus infectivity [213]. In particular, it has also been recently shown that cold plasmas may be used to selectively kill cancer cells without damaging surrounding healthy cells [214]. Kong et al. noted that the synergies between cold atmospheric plasmas and charged nanoparticles addressed the issues of reactivity, selectivity, toxicity and penetration required for effective drug delivery and medical treatment [208]. Also important to note is the issue of nanosafety. A recently published review on plasmas and nanosafety [215] suggests that plasma-based fabrication is a relatively safe method for the production of a range of nanomaterials [216].

\subsection{Some further links between plasmas and plasmons}

Let us now discuss more explicit links between plasma and plasmonic phenomena. Wang et al. [2] recently noted that the plasma screening effect (a fundamental, generic plasma property) leads to bandgap opening for bulk plasmons both in gaseous plasmas and metal plasmas. When an electromagnetic (EM) wave is incident on an interface between a plasma and a dielectric (e.g. vacuum), a charge displacement leads to the excitation of forward and backward plane waves. The screening happens below the plasma frequency when the plane waves destructively interfere with the incident EM wave creating an energy gap. Above the plasma frequency, the forward plane wave changes phase and constructively interfere with the incoming EM wave. This is now the transverse bulk plasmon [2] (see also the definition in Sect. 1.2). It should be noted that the bandgap is determined by the plasma density, i.e. the denser the plasma, the wider the bandgap. This property is very useful to determine (and tailor) the bandgap for specific types of propagating plasmons in materials.

Separating plasma-specific (e.g. energy absorption near plasmon resonance) and other (e.g., non-resonant) phenomena in plasmonic responses is also critical in optimising the performance of a range of devices, in particular, solar cells [185]. Comparison of silver (i.e. resonant in visible regime) and aluminium (i.e. non resonant in the visible regime) nanoparticles showed that they interact with incoming light differently. Aside from the "normal" scattering from nanoparticle geometric features including surface roughness, etc., resonant nanoparticles (i.e., silver) have the added complication of parasitic absorption around the plasmon resonance. This resonance, however, can be shifted by modifying the height:radius ratio. Depending on the nanoparticle material, and whether they are subject to any plasma-specific effects, the nanoparticle geometry will have to be modified to effect the best device performance. Moreover, study of plasmonic coupling, resonance frequencies etc. will facilitate plasma nanofabrication efforts to produce structures deterministically to optimize device response. Studying nanoscale plasma phenomena in plasmonic nanostructures should improve predictability of responses of nanophotonic devices which simultaneously use plasma and non-resonant optical effects. Although these studies in many cases require quantum treatment, they will still be able to draw on the physics of collective phenomena in gas plasmas as foundation.

Another analogy between plasmonics and plasma physics can be drawn, by examining electron-surface scattering. As discussed in Section 3, Mie scattering theory may be used to describe how nanoparticles of different sizes interact with and scatter light. However, we stress that this is a geometric effect, not a plasma effect. The scattering of electrons from a surface, causes a sizeintroduced collisionality in the plasma. This collisionality is included in the model for plasmonic nanoshells which 
incorporated an electron surface scattering term in the dielectric constant [84]:

$$
\varepsilon_{C}=\varepsilon_{e x p}-\frac{\omega_{p}^{2}}{\omega\left(\omega+i\left[\gamma_{\text {intra }}+\gamma\left(L_{B}\right)\right]\right)}+\frac{\omega_{p}^{2}}{\omega\left(\omega+i \gamma_{\text {intra }}\right)}
$$

where $\varepsilon_{\text {exp }}$ is the experimental dielectric constant, $\gamma=$ $\gamma_{\text {intra }}+\gamma\left(L_{B}\right)$ is the mean free path dependant damping, $\gamma\left(L_{B}\right)=V_{F} / L_{B}$ is the damping due to surface scattering where $V_{F}$ is the Fermi velocity, $L_{B}$ is the mean free path of the electrons in the cell and $\gamma_{\text {intra }}$ is the intraband damping term [84]. The size of the nanoparticles is important for $L_{B}$ which is equal to $\left(4\left(r_{0}^{3}-r_{i}^{3}\right)\right) /\left(3\left(r_{0}^{2}+r_{i}^{2}\right)\right)$, where $r_{0}$ and $r_{i}$ are the outer and inner radii, respectively. The specific material and geometry of the nanoparticle determine the contribution of surface scattering correction to dielectric permittivity [84]. For example, the surface scattering term is particularly important for thin shells causing a blue shift of the resonance. By taking this surface scattering term into account, one can tailor nanoarrays (and nanoplasmas) to achieve the resonant responses required.

A similar size-introduced collisionality effect is observed for discharge boundaries in gaseous microplasmas when the size of the discharge is reduced to submillimeter and even smaller dimensions [217]. The transition from a bulk plasma to a microplasma (typically at micrometre and submicrometre sizes) with size dependant properties occurs at a critical size determined by [217]:

- surface to volume ratio (SVR);

- electrode spacing (ES)

which is very similar to nanoplasmonic arrays.

As the SVR increases and ES decreases, the electron temperature and density increase, which in turn affects the collision rates in the plasma and hence the plasma characteristics. Similarly, scattering from the boundaries does affect collisionality and equilibrium of gaseous plasmas, although at different time scales and involving different species (atoms/molecules rather than electrons). Such discharges are still too large for electron scattering effects to become as important as in plasmonics. However, gaseous plasma discharges and plasma collective phenomena are strongly affected by secondary electron emission from the surface. It is presently unclear if this effect plays a role in plasmonics. Nevertheless, the similarity remains and could prove useful to note at a later juncture.

Moreover, in a contribution to the recently published 2012 Plasma Roadmap [218], Tachibana notes that microplasma arrays can be used just like a photonic crystal. They note by examining the permittivity [218]:

$$
\varepsilon_{p}=1-\frac{\omega_{p}^{2}}{\omega^{2}\left(1+i \nu_{m} / \omega\right)}
$$

where $\nu_{m}$ is the electron collision frequency, all other symbols defined as before, that the plasma density and the plasmon frequency $\left(\omega_{p}\right)$ is what will ultimately affect the response of the 'array'. Given a plasma density of around
$10^{16} \mathrm{~cm}^{-3}$, plasmonic responses could be expected in the as yet elusive $\mathrm{THz}$ range [218].

Another link between plasma physics and plasmonics is also possible - namely, using the emission from plasmas to excite plasmons. Using plasmas as an excitation source would potentially enable use of the same source to excite IR-VIS-UV plasmons requiring only simple tuning of the plasma parameters. It is an attractive research area - both in terms of the pure physics involved as well as applications. Thus it is an area that the authors of this colloquium are currently investigating and the results will be reported elsewhere.

It appears that as the fields of plasmonics and plasma nanoscience further develop there will be a likely common ground - not only in terms of fabrication and fundamental physics, but in real world, complementary applications in many fields. If an integrated approach which recognises the common physical foundations and similarities in the many physical phenomena is used, many new interesting effects may be discovered which in turn may lead to new applications in several fields.

\section{Conclusions and outlook for future synergistic research in the field}

To summarise, in this colloquium we have presented a slice of two quite distinct fields - plasma physics and plasmonics, connected by very similar physics, in particular, the notion of collective plasma waves and oscillations. We have presented the essential equations of each field and demonstrated their similarities and placed them firmly within the historical field. We then used the research field of Plasma Nanoscience to show that these similarities are not merely academic, but can be put into practice in the design of plasma-aided fabrication of nanoplasmonic arrays for a myriad of applications from sensors to photovoltaic cells. We also discussed emerging biomedical applications in both plasmonics and atmospheric plasmas which both contribute to the field of cancer treatment by recognising the link between plasmonics and plasmas in emergent fields such as cancer research, novel treatments and techniques using the best of both fields may be further developed. Indeed, as device design matures we will have increasing opportunity to study nanoplasmas and nanoplasma phenomena. Hence, by reaching back as far as primordial earth (for terrestrial gaseous plasmas) and the 4th century AD (for plasmons in solid plasmas) and looking forward to the state-of-the-art nanomedical and technological advances, one concludes that everything old, is ultimately, new once again.

A list of symbols and abbreviations used in the colloquium is provided in Table 2

A.E.R acknowledges support from the CSIRO OCE Postdoctoral Fellowship Program. This work was partially supported by the Australian Research Council, CSIRO OCE Science Leader Program and CSIRO Sensors \& Sensor network TCP. 


\section{References}

1. H.A. Atwater, A. Polman, Nat. Mater. 9, 205 (2010)

2. Y. Wang, E.W. Plummer, K. Kempa, Adv. Phys. 60, 799 (2011)

3. D.A. Gurnett, A. Bhattacharjee, Introduction to Plasma Physics: With Space and Laboratory Applications (Cambridge University Press, Cambridge, 2005)

4. A.L. Peratt, Astrophys. Space Sci. 242, 93 (1996)

5. K. Ostrikov, Phys. Plasmas 18, 057101 (2011)

6. K. Ostrikov, Rev. Mod. Phys. 77, 489 (2005)

7. K. Ostrikov, A.B. Murphy, J. Phys. D 40, 2223 (2007)

8. K. Willets, R.V. Duyne, Annu. Rev. Phys. Chem. 58, 267 (2007)

9. C.F. Bohren, D.R. Huffman, Absorption and Scattering of Light by Small Particles (John Wiley \& Sons, 1983)

10. D. Pines, Rev. Mod. Phys. 28, 184 (1956)

11. S. Maier, Plasmonics: Fundamentals and Applications (Springer, 2007)

12. D. Bohm, D. Pines, Phys. Rev. 82, 625 (1951)

13. D. Pines, D. Bohm, Phys. Rev. 85, 333 (1952)

14. D. Bohm, D. Pines, Phys. Rev. 92, 609 (1953)

15. M. Moisan, J. Margot, Z. Zakrzewski, in High Density Plasma Sources: Design, Physics and Performance, edited by O.A. Popov (Noyes Publications, 1995)

16. A.E. Miroshnichenko, S. Flach, Y.S. Kivshar, Rev. Mod. Phys. 82, 2257 (2010)

17. M. Faraday, Phil. Trans. R. Soc. Lond. 147, 145 (1857)

18. P. Drude, Ann. Phys. 1, 566 (1900)

19. G. Mie, Ann. Phys. 25, 377 (1908)

20. E. Kretschmann, H. Raether, Z. Naturforschung A 23, 2315 (1968)

21. A. Otto, Z. Physik 216, 398 (1968)

22. M. Fleischmann, P. Hendra, A. McQuillan, Chem. Phys. Lett. 26, 163 (1974)

23. G.M. Albrecht, J.A. Creighton, J. Am. Chem. Soc. 99, 5215 (1977)

24. D.L. Jeanmaire, R.P. van Duyne, J. Electroanal. Chem. 84, 120 (1977)

25. M.I. Stockman, L.N. Pandey, T.F. George, Phys. Rev. B 53, $2183(1996)$

26. S.M. Nie, S.R. Emery, Science 275, 1102 (1997)

27. T.W. Ebbesen, H.J. Lezec, H.F. Ghaemi, T. Thio, P.A. Wolff, Nature 391, 667 (1998)

28. R.H. Ritchie, Phys. Rev. 106, 874 (1957)

29. J. Zenneck, Ann. Phys. 328, 846 (1907)

30. A. Sommerfeld, Ann. Phys. 333, 665 (1909)

31. I. Langmuir, Proc. Natl. Acad. Sci. USA 14, 628 (1928)

32. L. Tonks, Phys. Rev. 38, 1219 (1931)

33. S. Ijima, Nature 354, 56 (1991)

34. K. Ostrikov, Plasma Nanoscience: Basic Concepts and Applications of Deterministic Nanofabrication (Wiley VCH, 2008)

35. L. Tonks, I. Langmuir, Phys. Rev. 33, 195 (1929)

36. J. Taillet, Am. J. Phys. 37, 423 (1969)

37. L. Tonks, Phys. Rev. 37, 1458 (1931)

38. D. Schumacher, C. Rea, D. Heitmann, K. Scharnberg, Surf. Sci. 408, 203 (1998)

39. U. Fano, Phys. Rev. 124, 1866 (1961)

40. B. Luk'yanchuk, N.I. Zheludev, S.A. Maier, N.J. Halas, P. Nordlander, H. Giessen, C.T. Chong, Nat. Mater. 9, 707 (2010)

41. M.I. Stockman, Phys. Today 64, 39 (2011)
42. E.L. Ru, M. Dalley, P. Etchegoin, Curr. Appl. Phys. 6, $411(2006)$

43. T.J. Davis, K.C. Vernon, D.E. Gomez, J. Appl. Phys. 106, 043502 (2009)

44. E. Castaños-Martínez, M. Moisan, Y. Kabouzi, J. Phys. D 42, 012003 (2009)

45. I. Ghanashev, H. Sugai, Plasma Sources Sci. Technol. 11, A178 (2002)

46. H. Sugai, I. Ghanashev, M. Nagatsu, Plasma Sources Sci. Technol. 7, 192 (1998)

47. Y. Tokunaga, H. Watanabe, A. Minamide, T. Minamikawa, Jpn J. Appl. Phys. 36 (Part 1), 3162 (1997)

48. R.J. Green, R.A. Frazier, K.M. Shakesheff, M.C. Davies, C.J. Roberts, S.J. Tendler, Biomaterials 21, 1823 (2000)

49. M. Chaker, M. Moisan, Z. Zakrzewski, Plasma Chem. Plasma Phys. 6, 79 (1986)

50. Y.A. Akimov, V.P. Olefir, N.A. Azarenkov, Contrib. Plasma Phys. 46, 817 (2006)

51. N. Azarenkov, I. Denisenko, K. Ostrikov, Surf. Rev. Lett. 2, 579 (1995)

52. K. Ostrikov, Phys. Scr. 60, 265 (1999)

53. K. Ostrikov, O. Osmayev, Surf. Sci. 344, 283 (1995)

54. N. Azarenkov, K. Ostrikov, Phys. Rep. 308, 333 (1999)

55. I. Ghanashev, M. Nagatsu, S. Morita, H. Sugai, J. Vac. Sci. Technol. A 16, 1537 (1998)

56. N. Azarenkov, I. Denisenko, K. Ostrikov, J. Phys. D 28, 2465 (1995)

57. K. Ostrikov, M. Yu, N. Azarenkov, J. Appl. Phys. 84, 4176 (1998)

58. E. Castaños-Martínez, M. Moisan, IEEE Trans. Plasma Sci. 39, 2192 (2011)

59. E. Castaños-Martínez, M. Moisan, Expansion of Contracted Single Rare-Gas Tubular Discharges at Atmospheric Pressure, in 52nd Annual Technical Conference Proceedings, Santa Clara, CA (2009), p. 333, ISSN 0737-592

60. J. Wolinska-Szatkowska, J. Phys. D 21, 937 (1988)

61. H.J. Lee, Plasma Phys. Contr. F 46, 447 (2004)

62. E. Tatarova, F.M. Dias, J. Henriques, C.M. Ferreira, J. Phys. D 39, 2747 (2006)

63. D.J. Cooperberg, C.K. Birdsall, Plasma Sources Sci. Technol. 7, 41 (1998)

64. I. Ghanashev, M. Nagatsu, H. Sugai, Jpn J. Appl. Phys. 36, 337 (1997)

65. I. Ghanashev, M. Nagatsu, G. Xu, H. Sugai, Jpn J. Appl. Phys. 36, 4704 (1997)

66. T. Ishijima, H. Toyoda, Y. Takanishi, H. Sugai, Jpn J. Appl. Phys. 50, 036002 (2011)

67. M. Nagatsu, I. Ghanashev, S. Morita, H. Sugai, Jpn J. Appl. Phys. 37, 2406 (1998)

68. M. Nagatsu, S. Morita, I. Ghanashev, A. Ito, N. Toyoda, H. Sugai, J. Phys. D 33, 1143 (2000)

69. C.M. Ferreira, E. Tatarova, J. Henriques, F.M. Dias, J. Phys. D 42, 194016 (2009)

70. S.Y. Choi, J.M. White, Q. Wang, L. Hou, K.W. Kim, S. Kurita, T.K. Won, S. Anwar, B.S. Park, R.L. Tiner, Plasma uniformity control by gas diffuser hole design (2011), http://www.freepatentsonline.com/y2011/ 0290183.html, US Patent Application 20050251990

71. A.V. Zayats, I.I. Smolyaninov, A.A. Maradudin, Phys. Rept. 408, 131 (2005)

72. K.M. Mayer, J.H. Hafner, Chem. Rev. 111, 3828 (2011) 
73. R. Gans, Ann. Phys. 342, 881 (1912)

74. R. Gans, Ann. Phys. 352, 270 (1915)

75. E. Prodan, C. Radloff, N.J. Halas, P. Nordlander, Science 302, 419 (2003)

76. H. Wang, D.W. Brandl, F. Le, P. Nordlander, N.J. Halas, Nano Lett. 6, 827 (2006)

77. M.D. Turner, M.M. Hossain, M. Gu, New J. Phys. 12, $083062(2010)$

78. M.E. Stewart, C.R. Anderton, L.B. Thompson, J. Maria, S.K. Gray, J.A. Rogers, R.G. Nuzzo, Chem. Rev. 108, $494(2008)$

79. P.R. West, S. Ishii, G.V. Naik, N.K. Emani, V.M. Shalaev, A. Boltasseva, Laser Photon. Rev. 4, 795808 (2010)

80. M. Rycenga, C.M. Cobley, J. Zeng, W. Li, C.H. Moran, Q. Zhang, D. Qin, Y. Xia, Chem. Rev. 111, 3669 (2011)

81. M.G. Blaber, M.D. Arnold, M.J. Ford, J. Phys.: Condens. Matter 22, 143201 (2010)

82. B. de Bas, M. Ford, M. Cortie, J. Phys.: Condens. Matter 18, 55 (2006)

83. M.G. Blaber, M.D. Arnold, M.J. Ford, J. Phys.: Condens. Matter 22, 095501 (2010)

84. M.G. Blaber, M.D. Arnold, M.J. Ford, J. Phys. Chem. C 113, 3041 (2009)

85. M.G. Blaber, M.D. Arnold, M.J. Ford, J. Phys.: Condens. Matter 21, 144211 (2009)

86. M.G. Blaber, M.D. Arnold, N. Harris, M.J. Ford, M.B. Cortie, Physica B 394, 184 (2007)

87. J. Heber, Nature 461, 720 (2009)

88. K.R. Catchpole, S. Pillai, J. Lumin. 121, 315 (2006)

89. K.R. Catchpole, S. Pillai, J. Appl. Phys. 100, 044504 (2006)

90. S. Pillai, F.J. Beck, K.R. Catchpole, Z. Ouyang, M.A. Green, J. Appl. Phys. 109, 073105 (2011)

91. A.M. Funston, C. Novo, T.J. Davis, P. Mulvaney, Nano Lett. 9, 1651 (2009)

92. N. Harris, M.J. Ford, P. Mulvaney, M.B. Cortie, Gold Bull. 41, 5 (2008)

93. P.K. Jain, W. Huang, M.A. El-Sayed, Nano Lett. 7, 2080 (2007)

94. M.I. Stockman, Opt. Express 19, 22029 (2011)

95. S.M. Stranahan, K.A. Willets, Nano Lett. 10, 3777 (2010)

96. M.L. Weber, K.A. Willets, J. Phys. Chem. Lett. 2, 1766 (2011)

97. D.E. Gomez, K.C. Vernon, T.J. Davis, Phys. Rev. B 81 $075414(2010)$

98. D.E. Gomez, K.C. Vernon, P. Mulvaney, T.J. Davis, Appl. Phys. Lett. 96, 073108 (2010)

99. D.E. Gomez, K.C. Vernon, P. Mulvaney, T.J. Davis, Nano Lett. 10, 274 (2010)

100. K. Aydin, I.M. Pryce, H.A. Atwater, Opt. Express 18, 13407 (2010)

101. A.L. Baudrion, F. de Leon-Perez, O. Mahboub, A. Hohenau, H. Ditlbacher, F.J. Garcia-Vidal, J. Dintinger, T.W. Ebbesen, L. Martin-Moreno, J.R. Krenn, Opt. Express 16, 3420 (2008)

102. J.M. Bingham, K.A. Willets, N.C. Shah, D.Q. Andrews, R.P. Van Duyne, J. Phys. Chem. C 113, 16839 (2009)

103. R.M. Briggs, J. Grandidier, S.P. Burgos, E. Feigenbaum, H.A. Atwater, Nano Lett. 10, 4851 (2010)

104. T.J. Davis, D.E. Gomez, K.C. Vernon, Phys. Rev. B 82, $205434(2010)$
105. T.J. Davis, K.C. Vernon, D.E. Gomez, Phys. Rev. B 79, $155423(2009)$

106. V.E. Ferry, L.A. Sweatlock, D. Pacifici, H.A. Atwater, Nano Lett. 8, 4391 (2008)

107. L.A. Sweatlock, S.A. Maier, H.A. Atwater, J.J. Penninkhof, A. Polman, Phys. Rev. B 71, 235408 (2005)

108. K. Ostrikov, J. Phys. D 44, 174003 (2011)

109. K. Ostrikov, I. Levchenko, S. Xu, Pure. Appl. Chem. 80, 1909 (2008)

110. K. Ostrikov, U. Cvelbar, A.B. Murphy, J. Phys. D 44, 174001 (2011)

111. K. Ostrikov, I. Levchenko, U. Cvelbar, M. Sunkara, M. Mozetic, Nanoscale 2, 2012 (2010)

112. K. Ostrikov, D.H. Seo, H. Mehdipour, Q. Cheng, S. Kumar, Nanoscale 4, 1497 (2012)

113. A.E. Rider, K. Ostrikov, Plasma Processing of Nanomaterials, in Nanomaterials and their Applications, edited by R.M. Sankaran (CRC Press, Boca Raton, USA, 2011), Chap. 15, pp. 371-392

114. M. Meyyappan, J. Phys. D 44, 174002 (2011)

115. A. Anders, J. Phys. D 40, 2272 (2007)

116. A.E. Rider, K. Ostrikov, I. Levchenko, Nanotechnology 19, 355705 (2008)

117. A. Dvurechenskii, J. Smagina, R. Groetzschel, V. Zinovyev, V. Armbrister, P. Novikov, S. Teys, A. Gutakovskii, Surf. Coat. Technol. 196, 25 (2005)

118. A.E. Rider, I. Levchenko, K. Ostrikov, M. Keidar, Plasma Process. Polym. 4, 638 (2007)

119. J.C. Ho, I. Levchenko, K. Ostrikov, J. Appl. Phys. 101, 094309 (2007)

120. I. Levchenko, K. Ostrikov, D. Mariotti, Carbon 47, 344 (2009)

121. Z. Tsakadze, K. Ostrikov, S. Xu, Surf. Coat. Technol. 191, 49 (2005)

122. X. Landreau, B. Lanfant, T. Merle, C. Laborde, E. Dublanche-Tixier, P. Tristant, Eur. Phys. J. D 65, 421 (2011)

123. Y.J. Hung, S.L. Lee, B. Thibeault, L. Coldren, IEEE J. Sel. Top. Quant. 17, 869 (2011)

124. I. Levchenko, K. Ostrikov, M. Keidar, S. Xu, Appl. Phys. Lett. 89, 033109 (2006)

125. L. Yuan, X. Zhong, I. Levchenko, Y. Xia, K. Ostrikov, Plasma Proc. Polym. 4, 612 (2007)

126. X. Zhong, X. Wu, K. Ostrikov, Plasma Proc. Polym. 6, $161(2009)$

127. L. Yuan, X. Zhong, K. Ostrikov, Nanotechnology 19, 155304 (2008)

128. H. Kersten, H. Deutsch, H. Steffen, G.M.W. Kroesen, R. Hippler, Vacuum 63, 385 (2001)

129. H. Kersten, D. Rohde, H. Steffen, H. Deutsch, R. Hippler, G.H.P.M. Swinkels, G.M.W. Kroesen, Appl. Phys. A 72, $531(2001)$

130. D. Rohde, P. Pecher, H. Kersten, W. Jacob, R. Hippler, Surf. Coat. Technol. 149, 206 (2002)

131. J. Blazek, P. Bartos, R. Basner, H. Kersten, P. Spatenka, Eur. Phys. J. D 54, 219 (2009)

132. G. Schubert, R. Basner, H. Kersten, H. Fehske, Eur. Phys. J. D 63, 431 (2011)

133. R.E. Bailey, S. Nie, J. Am. Chem. Soc. 125, 7100 (2003)

134. A.E. Rider, I. Levchenko, K. Ostrikov, J. Appl. Phys. 101, 044306 (2007)

135. A.E. Rider, K. Ostrikov, Surf. Sci. 603, 359 (2009) 
136. Q. Cheng, S. Xu, J.D. Long, Z.H. Ni, A.E. Rider, K. Ostrikov, J. Phys. D 41, 055406 (2008)

137. Q.J. Cheng, J.D. Long, Z. Chen, S. Xu, J. Phys. D 40, $2304(2007)$

138. M. Xu, S. Xu, J.W. Chai, J.D. Long, Q.J. Cheng, Y.C. Ee, K. Ostrikov, J. Appl. Phys. 103, 053512 (2008)

139. Q.J. Cheng, J.D. Long, S. Xu, J. Appl. Phys. 101, 094304 (2007)

140. A. Das Arulsamy, A.E. Rider, Q.J. Cheng, S. Xu, K. Ostrikov, J. Appl. Phys. 105, 094314 (2009)

141. W.H. Chiang, R. Mohan Sankaran, Nat. Mater. 8, 882 (2009)

142. K. Kempa, Phys. Rev. B 66, 195406 (2002)

143. L. Ju et al., Nat. Nanotechol. 6, 630 (2011)

144. M. Jablan, H. Buljan, M. Soljacic, Phys. Rev. B 80, $245435(2009)$

145. F.H.L. Koppens, D.E. Chang, F. Javier Garcia de Abajo, Nano Lett. 11, 3370 (2011)

146. N.L. Rangel, J.M. Seminario, J. Chem. Phys. 132, 125102 (2010)

147. W. Wang, P. Apell, J. Kinaret, Phys. Rev. B 84, 085423 (2011)

148. A.E. Rider, S. Kumar, S.A. Furman, K. Ostrikov, Chem. Commun. 48, 2659 (2012)

149. A. Bostwick, F. Speck, T. Seyller, K. Horn, M. Polini, R. Asgari, A.H. MacDonald, E. Rotenberg, Science 328, 999 (2010)

150. S. Thongrattanasiri, A. Manjavacas, F.J. García de Abajo, ACS Nano 6, 1766 (2012)

151. J. Christensen, A. Manjavacas, S. Thongrattanasiri, F.H.L. Koppens, F.J. García de Abajo, ACS Nano 6, 431440 (2012)

152. F.H.L. Koppens, D.E. Chang, F. Javier Garcia de Abajo, Nano Lett. 11, 3370 (2011)

153. D. Else, R. Kompaneets, S.V. Vladimirov, Europhys. Lett. 94, 35001 (2011)

154. A. Mushtaq, S.V. Vladimirov, Eur. Phys. J. D 64, 419 (2011)

155. Y. Tyshetskiy, S.V. Vladimirov, R. Kompaneets, Phys. Plasmas 18, 12104 (2011)

156. S.V. Vladimirov, Plasma Phys. Contr. F 53, 074005 (2011)

157. D.V. Fisher, Y. Maron, Eur. Phys. J. D 18, 93 (2002)

158. F. Haas, Europhys. Lett. 77, 45004 (2007)

159. G.A. Hoshoudy, Plasma Phys. Rep. 37, 775 (2011)

160. D.H. Ki, Y.D. Jung, Europhys. Lett. 91, 65001 (2010)

161. A.P. Misra, N.K. Ghosh, C. Bhowmik, Eur. Phys. J. D 49, 373 (2008)

162. A. Schmidt-Bleker, W. Gassen, H. Kull, Europhys. Lett. 95, 55003 (2011)

163. N.L. Tsintsadze, L.N. Tsintsadze, A. Hussain, G. Murtaza, Eur. Phys. J. D 64, 447 (2011)

164. N.J.M. Horing, Phil. Trans. R. Soc. A 368, 5525 (2010)

165. N.J.M. Horing, Phys. Rev. B 80, 193401 (2009)

166. N.J.M. Horing, Introduction to Complex Plasmas, in Springer Series on Atomic, Optical, and Plasma Physics, edited by M. Bonitz, N. Horing, P. Ludwig (Springer Berlin Heidelberg, 2010), Vol. 59, pp. 109-132

167. D.H. Seo, S. Kumar, K. Ostrikov, J. Mater. Chem. 21, 16339 (2011)

168. P.A. Huidobro, M.L. Nesterov, L. MartÃn-Moreno, F.J. García-Vidal, New J. Phys. 13, 033011 (2011)
169. H. Cang, A. Labno, C. Lu, X. Yin, M. Liu, C. Gladden, Y. Liu, X. Zhang, Nature 469, 385 (2011)

170. R. Bardhan, S. Lal, A. Joshi, N.J. Halas, Acc. Chem. Res. 44, 936 (2011)

171. N. Liu, M. Hentschel, T. Weiss, A.P. Alivisatos, H. Giessen, Science 332, 1407 (2011)

172. P. Neuzil, J. Reboud, Anal. Chem. 80, 6100 (2008)

173. M.A. Noginov, G. Zhu, A.M. Belgrave, R. Bakker, V.M. Shalaev, E.E. Narimanov, S. Stout, E. Herz, T. Suteewong, U. Wiesner, Nature 460, 1110 (2009)

174. P.A. Huidobro, M.L. Nesterov, L. Martiİn-Moreno, F.J. Garciİa-Vidal, Nano Lett. 10, 1985 (2010)

175. G. Adamo, Plasmons transform, Optics \& Photonics Focus 10, Story 6 - 20/9/2010 (2010), http://opfocus. org/index $\cdot$ php?topic $=$ story\&v $=10 \& s=6$

176. D.S. Wiersma, M.A. Noginov, J. Opt. 12, 020201 (2010)

177. M.A. Noginov, G. Zhu, M. Mayy, B.A. Ritzo, N. Noginova, V.A. Podolskiy, Phys. Rev. Lett. 101, 226806 (2008)

178. Y.A. Akimov, W.S. Koh, S.Y. Sian, S. Ren, Appl. Phys. Lett. 96, 073111 (2010)

179. F.J. Beck, A. Polman, K.R. Catchpole, J. Appl. Phys. 105, 114310 (2009)

180. K.R. Catchpole, A. Polman, Appl. Phys. Lett. 93, 191113 (2008)

181. K.R. Catchpole, A. Polman, Photovoltaic cell with surface plasmon resonance generating nano-structures (2009), http://www.patentstorm.us/applications/ 20110197959/claims.html, US Patent Application 20110197959

182. K.R. Catchpole, A. Polman, Opt. Express 16, 21793 (2008)

183. Y.A. Akimov, K. Ostrikov, E.P. Li, Plasmonics 4, 107 (2009)

184. Y. Akimov, W. Koh, K. Ostrikov, Opt. Express 17, 10195 (2009)

185. Y. Akimov, W. Koh, Plasmonics 6, 155 (2011)

186. I. Levchenko, K. Ostrikov, A.B. Murphy, J. Phys. D 41, 092001 (2008)

187. M.A. Green, S. Pillai, Nat. Photon. 6, 130 (2012)

188. Y. Wang, T. Sun, T. Paudel, Y. Zhang, Z. Ren, K. Kempa, Nano Lett. 12, 440 (2012)

189. L. Wu, H.S. Chu, W.S. Koh, E.P. Li, Opt. Express 18, 14395 (2010)

190. D.J. O'Shannessy, M. Brigham-Burke, K. Peck, Anal. Biochem. 205, 132 (1992)

191. R.J. Fisher, A. Rein, M. Fivash, M.A. Urbaneja, J.R. Casas-Finet, M. Medaglia, L.E. Henderson, J. Virol. 72, 1902 (1998)

192. R. Morrow, D. McKenzie, M. Bilek, C. MacDonald, M. Stindt, G. Anetsberger, A. Martin, Physica B 394, 203 (2007)

193. B.N. Feltis, B.A. Sexton, F.L. Glenn, M.J. Best, M. Wilkins, T.J. Davis, Biosens. Bioelectron. 23, 1131 (2008)

194. N. Harris, M.D. Arnold, M.G. Blaber, M.J. Ford, J. Phys. Chem. C 113, 2784 (2009)

195. T.J. Davis, D.E. Gomez, K.C. Vernon, Nano Lett. 10, 2618 (2010)

196. T.J. Davis, K.C. Vernon, D.E. Gomez, Opt. Express 17, 23655 (2009)

197. K.C. Vernon, A.M. Funston, C. Novo, D.E. Gomez, P. Mulvaney, T.J. Davis, Nano Lett. 10, 2080 (2010) 
198. K.L. Lee, S.H. Wu, C.W. Lee, P.K. Wei, Opt. Express 19, 24530 (2011)

199. N.R. Agarwal, E. Fazio, F. Neri, S. Trusso, C. Castiglioni, A. Lucotti, N. Santo, P.M. Ossi, Cryst. Res. Technol. 46, $836(2011)$

200. K.C. Vernon, T.J. Davis, F.H. Scholes, D.E. Gomez, D. Lau, J. Raman Spectrosc. 41, 1106 (2010)

201. E.S. Shibu, J. Cyriac, T. Pradeep, J. Chakrabarti, Nanoscale 3, 1066 (2011)

202. S.A. Maier, Opt. Express 14, 1957 (2006)

203. D. Pissuwan, S.M. Valenzuela, M.C. Killingsworth, X. Xu, M.B. Cortie, J. Nanopart. Res. 9, 1109 (2007)

204. F. Ratto, P. Matteini, S. Centi, F. Rossi, R. Pini, J. Biophotonics 4, 64 (2011)

205. P.K. Jain, I.H. El-Sayed, M.A. El-Sayed, Nano Today 2, $18(2007)$

206. J.Z. Zhang, J. Phys. Chem. Lett. 1, 686 (2010)

207. N.M. Schaeublin, L.K. Braydich-Stolle, A.M. Schrand, J.M. Miller, J. Hutchison, J.J. Schlager, S.M. Hussain, Nanoscale 3, 410 (2011)

208. M.G. Kong, M. Keidar, K. Ostrikov, J. Phys. D 44, 174018 (2011)

209. K.D. Weltmann, E. Kindel, R. Brandenburg, C. Meyer, R. Bussiahn, C. Wilke, T. von Woedt ke, Contrib. Plasma Phys. 49, 631 (2009)

210. G. Daeschlein, S. Scholz, A. Arnold, T. von Woedtke, E. Kindel, M. Niggemeier, K.D. Weltmann, M. Jünger, IEEE Trans. Plasma Sci. 38, 2969 (2010)

211. S. Rupf, A. Lehmann, M. Hannig, B. Schäfer, A. Schubert, U. Feldmann, A. Schindler, J. Med. Microbiol. 59, 206 (2010)
212. J. Huang, H. Li, W. Chen, G.H. Lv, X.Q. Wang, G.P. Zhang, K. Ostrikov, P.Y. Wang, S.Z. Yang, Appl. Phys. Lett. 99, 253701 (2011)

213. Z. Xiong, X. Lu, Y. Cao, Q. Ning, K. Ostrikov, Y. Lu, X. Zhou, J. Liu, Appl. Phys. Lett. 99, 253703 (2011)

214. M. Keidar, R. Walk, A. Shashurin, P. Srinivasan, A. Sandler, S. Dasgupta, R. Ravi, R. Guerrero-Preston, B. Trink, Br. J. Cancer 105, 1295 (2011)

215. Z.J. Han, I. Levchenko, S. Kumar, M.M.A. Yajadda, S. Yick, D.H. Seo, P.J. Martin, S. Peel, Z. Kuncic, K. Ostrikov, J. Phys. D 44, 174019 (2011)

216. C. Fisher, A.E. Rider, S. Kumar, I. Levchenko, Z. Han, K. Ostrikov, J. Nanomater. 2012, 315185 (2012)

217. D. Mariotti, R.M. Sankaran, J. Phys. D 43, 323001 (2010)

218. S. Samukawa et al., J. Phys. D 45, 253001 (2012)

219. J.M. Pitarke, V.M. Silkin, E.V. Chulkov, P.M. Echenique, Rep. Prog. Phys. 70, 1 (2007)

220. S.Y. Shin, N.D. Kim, J.G. Kim, K.S. Kim, D.Y. Noh, K.S. Kim, J.W. Chung, Appl. Phys. Lett. 99, 082110 (2011)

221. C. Tegenkamp, H. Pfnür, T. Langer, J. Baringhaus, H. Schumacher, J. Phys.: Condens. Matter 23, 012001 (2011)

Open Access This is an open access article distributed under the terms of the Creative Commons Attribution License (http://creativecommons.org/licenses/by/3.0), which permits unrestricted use, distribution, and reproduction in any medium, provided the original work is properly cited. 